\title{
A Dynamical Modelling of the Epidemic Diseases to Assessing the Rates of Spread of COVID-19 in Saudi Arabia: SEIQR Model
}

Hamdy Youssef ( $\sim$ youssefanne2005@gmail.com )

Umm Al-Qura University https://orcid.org/0000-0003-4919-2966

Najat Alghamdi

Umm Al-Qura University

Magdy Ezzat

Al-Qassim University

Alaa El-Bary

Arab Academy for Science, Technology and Maritime Transport

Ahmed Shawky

Umm Al-Qura University

\section{Research Article}

Keywords: COVID-19, Jacobian matrix, Lyapunov stability, Novel coronavirus, Reproduction number, SEIR model, SEIRQ model

Posted Date: December 18th, 2020

DOl: https://doi.org/10.21203/rs.3.rs-77792/v2

License: (c) (i) This work is licensed under a Creative Commons Attribution 4.0 International License.

Read Full License 


\title{
A Dynamical Modelling of the Epidemic Diseases to Assessing the Rates of Spread of COVID-19 in Saudi Arabia: SEIQR Model
}

Hamdy Youssef ${ }^{1 *}$, Najat Alghamdi², Magdy A. Ezzat ${ }^{3}$, Alaa A. El-Bary ${ }^{4}$, and Ahmed M. Shawky 5

${ }^{1}$ Mechanical Engineering Department, College of Engineering and Islamic Architecture, Umm Al-Qura University, Makkah, Saudi Arabia.

*Corresponding Author: youssefanne2005@gmail.com \& hmyoussef@uqu.edu.sa

${ }^{2}$ Department of Mathematics, Faculty of Applied Science, Umm Al-Qura University, Makkah, Saudi Arabia; najatalghamdi@gmail.com \& naghamdi@uqu.edu.sa

${ }^{3}$ College of Science and Arts, Al-Qassim University, Al Bukairiyah, Al Qassim, Saudi Arabia; maezzat2000@yahoo.com

${ }^{4}$ Basic and Applied Science Institute, Arab Academy for Science, Technology and Maritime Transport, P.O. Box 1029, Alexandria, Egypt; aaelbary@ aast.edu

${ }^{5}$ Science and Technology Unit (STU), Umm Al-Qura University, Makkah, Saudi Arabia; amesmail@uqu.edu.sa

\begin{abstract}
A model of critical epidemic dynamics for the emergence of the new coronavirus COVID-19 is being established in this paper. A new approach to the assessment and control of the COVID-19 epidemic is given with the SEIQR pandemic model. This paper uses real knowledge on the distribution of COVID-19 in Saudi Arabia for mathematical modeling and dynamic analyses. The reproductive number and detailed stability analysis are provided in the SEIQR model dynamics. In a Jacobian method of linearization, we will address the domain of the solution and the equilibrium situation based on the SEIQR model. The equilibrium and its
\end{abstract}


importance have been proven, and a study of the stability of the equilibrium free from diseases has been implemented. The reproduction number was evaluated in accordance with its internal parameters. The Lyapunov theorem of stability has proven the global stability of the current model's equilibrium. The SEIQR model was contrasted by comparing the results based on the SEIQR model with the real COVID-19 spread data in Saudi Arabia. Numerical evaluation and predictions were given. The results indicate that the SEIQR model is a strong model for the study of the spread of epidemics, such as COVID-19. At the end of this work, we implemented an optimum protocol that can quickly stop the spread of COVID-19 among the Saudi populations. The key solution to slowing COVID-19 transmission is to stay home and bring sick persons as far as possible in a remote location or in a safe place. Ultimately, it is vital to offer safe and adequate treatment to ill people, and to avoid them, medications, tones, and nutrients should be provided to non-infected persons.

Keywords: COVID-19, Jacobian matrix, Lyapunov stability, Novel coronavirus, Reproduction number, SEIR model, SEIQR model

\section{Introduction}

As COVID-19 outbreaks continue, the number of infections steadily increases. This is due to the presence of many factors that increase the complexities of COVID-19 infection and create barriers to disease management. Since scientists and researchers all over the world are trying to establish a vaccine or a cure for the outbreak to control such pandemics in the future, from a medical engineering framework, an infectious disease can be well known and understood through the use of mathematical models. This idea began in 1927. After that, many different mathematical models have been constructed for various diseases and infections. For some essential studies, we refer to ${ }^{1-8}$. 
To explain transmission dynamics and estimate domestic and global disease spread based on data recorded from December 31,2019 , to January 28,2020 , Wu et al. ${ }^{9}$ have implemented the Susceptible Exposed Infectious Recovered Model (SEIR). They also found that COVID-19 had a fundamental reproductive number of approximately 2.68. Read et al. ${ }^{10}$ registered a value of 3.1 for the fundamental reproductive number based on the SEIR model data adaptation, assuming that the daily time spent by Poisson increases. Tang et al. ${ }^{11}$ suggested a deterministic compartmental model that included clinical disease progression, individual epidemiological status, and intervention steps. The authors found that the number of reproductive controls can be up to 6.47 and that engagement techniques, such as simplified traceability accompanied by insulation and quarantine, may minimize reproductive control numbers and risk of transmission effectively.

To determine the scale of the disease outbreak in Wuhan, $\operatorname{Iman}^{12}$ carried out calculational modeling of the possible epidemic tracks with an emphasis on human-to-human transmissions. Its findings suggest that controls must be efficiently controlled by well over $60 \%$ of the transmission. To analyze and forecast the infectivity of the new coronavirus, Guo et al. ${ }^{13}$ developed a deep learning algorithm. They found that two animal hosts of this virus were bats and minks. Most of the models illustrate the significant role of a direct transmission mechanism between humans and humans in the outbreak, as demonstrated by the fact that many individuals infected in the Wuhan area have no interaction and the number of infections has been growing rapidly and spreading across the Chinese provinces and over 20 people ${ }^{14}$. There is a relatively long incubation period in many infected individuals, so they do not show symptoms and have not been aware of their infection for 10-14 days. Over time, the disease can easily be spread by direct exposure to other people. On the other hand, the published models have not, to date, taken into account the environmental position of COVID-19 transmission. Several other modeling studies for the COVID-19 outbreak have also been carried out ${ }^{6,8,15-23}$. 
Statistical epidemiology is based on the dynamics of health and disease and related population factors. The presence of a pathogenic microbial agent identifies an infectious disease as a clinically obvious disease. For modeling purposes, four forms of transmission are characterized: straightforward, if the causative disease agent is individual; vector, if the causative agent is transmitted from a vector to a person; natural, if the touch of a pathogen infects the human via the environment; and vertical, if the disease agent is transmitted from mother to child at birth. Airborne and personal diseases are generally known to be transmitted directly where transmission occurs through contact between individuals and others. ${ }^{24}$.

Mathematical modeling of infectious diseases is important and critical with the advent of HIV epidemics. Since then, several models for investigating infectious diseases have been developed, studied, and applied. Mathematical modeling currently applies enormously to public health and mathematics. ${ }^{3,15,18,23,25}$.

In the emergence of HIV epidemics, the mathematical modeling of infectious diseases is significant. Since then, numerous models have been developed, studied, and applied for the investigation of infectious diseases ${ }^{26}$. Farman et al. studied the stability and control of glucose insulin glucagon system in human ${ }^{27}$. Farman et al. ${ }^{26}$ discussed the dynamical behavior of fractional-order cancer model with vaccine strategy. Gondim and Machado ${ }^{28}$ introduced the optimal quarantine strategies for the COVID-19 pandemic in a population with a discrete age structure. Davies et al. ${ }^{29}$ studied the age-dependent effects in the transmission and control of COVID-19 epidemics.

The goal of this paper is to construct a new COVID-19 vital dynamical model that is more applicable to cases in any country through mathematical analysis of the model in question by using a system of similar models with different considerations and new in / outflows between population divisions. In addition, this paper presents a new formula that explores the sensitivity of a reproduction number. The mechanisms of virus transmission by humans are to be 
discovered. Another aim is to investigate and learn the optimal procedures, controls, and techniques to minimize the outbreak substantially.

\section{Materials and Methods}

\subsection{Formulation of a coronavirus disease (SEIQR model)}

During the spread of COVID-19 in any country, the population can be divided into five vital dynamic subpopulations or five groups, which are represented in Fig. 1 and can be described as follows $4,6,16,23,29-31$ :

The main group $S(t)$ is dedicated to the healthy people but who may get the disease population. For certain diseases, the infected person may not become infectious immediately, but the latent phase is not contagious. It takes time for the pathogen to replicate and develop itself in the new host. In general, the exposed (latent) cycle follows the sensitive process $4,6,18,20,30$

Thus, the group $E(t)$ is dedicated to the exposed population or individuals who are infected but not yet infectious.

The group $I(t)$ is devoted to the population who are confirmed infected (individuals who have contracted the disease and are now sick with it and infected individuals are also infectious).

The group $Q(t)$ is dedicated to the quarantined population (separated from the general population even in their houses).

The group $R(t)$ is defined as the recovered population (individuals who have recovered and cannot contract the COVID-19 again), as in Fig. 1. 


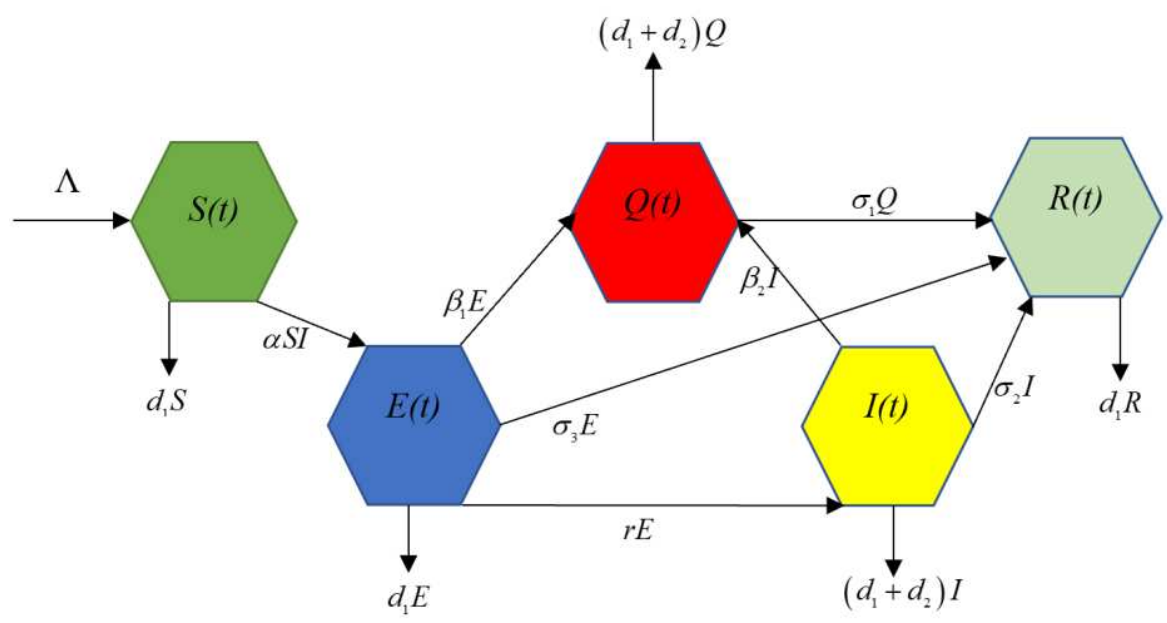

Figure 1. The flowchart of the SEIQR model

The parameter $\alpha$ is defined as the transmission rate from a susceptible population to infected but not detected by the testing population. We consider the net inflow of the susceptible population at a non-negative rate $\Lambda>0$ per unit value of time (comprising new births and new residents).

For any group, the outflow based on the natural death rate is defined by the nonnegative rate $d_{1}$.

The total population size is $N(t)$, which is defined as $4,6,16,22,23,25,32$ :

$$
N(t)=S(t)+E(t)+I(t)+R(t)+Q(t)
$$

Starting with group $S(t)$, we have two outflows; a population flows out to the exposed group $E(t)$ by the rate $\alpha I(t)$ (each one in $S(t)$ can transfer the infection to $\alpha I(t)$ ), so the total number of outflows is equal to multiple $\alpha S(t) I(t)$ ), and the outflow of the natural death is $d_{1} S$.

The group of exposed $E(t)$ has only one inflow $\alpha I(t) S(t)$, while it has four outflows. The first outflow is the population that flows out to group $Q(t)$ by the rate of transmission $\beta_{1}$ . The second outflow is the population that flows out to the recovery group directly without needing treatment by transmission rate of recovery $\sigma_{3}$. The third outflow is a population that 
flows out to the infected group $I(t)$ with the transmission rate of infected $r$, and the fourth outflow is the population that experiences natural death by the transmission rate $d_{1}^{4,6,16,22,23,25,32}$.

For the group of confirmed infected population $I(t)$, we have only one inflow, which comes from the group $E(t)$, with the transmission rate $r$, while it has three outflows of population. The first outflow is the population that must go to the quarantine area $Q(t)$ by the transmission rate $\beta_{2}$, and the second outflow comes from the population in which treatment has succeeded; individuals in this population can go out to the recovery group $R(t)$ by recovery transmission rate $\sigma_{2}$. The last outflow from the infected group is the total death, which comes from natural death by transmission rate $d_{1}$ and death due to the COVID-19 virus by transmission rate of mortality $d_{2}$.

For the recovery population $R(t)$, three inflows exist, and only one outflow. The first inflow comes from the quarantine area $Q(t)$ by transmission rate of recovery $\sigma_{1}$, the second inflow is the population that comes out from the infected group by transmission recovery rate $\sigma_{2}$, and the third inflow is the population that flows out from the exposed area directly by transmission recovery rate $\sigma_{3}$. The only outflow from the recovering group is death by the natural transmission rate of mortality $d_{1}$.

For the quarantine group $Q(t)$, two inflows $\beta_{1} E(t)$ and $\beta_{2} I(t)$ two outflows are present. The first outflow is the population flow out to the recovery group $R(t)$ with transmission rate $\sigma_{1}$, while the second outflow is the total death, which comes from natural death by transmission rate of death $d_{1}$ and by the transmission rate of death due to the COVID-19 virus $d_{2}$. 
All inflows and outflows are shown in the flowchart in Fig. 1, and the five groups can be converted into equations to formulate the following system of first-order ordinary nonlinear differential equations $4,6,16,22,23,25,32$ :

$$
\begin{gathered}
\frac{d S(t)}{d t}=\Lambda-\alpha S(t) I(t)-d_{1} S(t) \\
\frac{d E(t)}{d t}=\alpha S(t) I(t)-\varepsilon_{1} E(t) \\
\frac{d I(t)}{d t}=r E(t)-\varepsilon_{2} I(t) \\
\frac{d R(t)}{d t}=\sigma_{3} E(t)+\sigma_{2} I(t)-d_{1} R(t)+\sigma_{1} Q(t) \\
\frac{d Q(t)}{d t}=\beta_{1} E(t)+\beta_{2} I(t)-\varepsilon_{3} Q(t)
\end{gathered}
$$

where $\varepsilon_{1}=\left(r+\beta_{1}+\sigma_{3}+d_{1}\right), \varepsilon_{2}=\left(\beta_{2}+\sigma_{2}+d_{1}+d_{2}\right)$, and $\varepsilon_{3}=\left(\sigma_{1}+d_{1}+d_{2}\right)$.

2.2 Theorem 1 (all solutions are definite positive)

Let the initial conditions $\{S(0), E(0), I(0), Q(0), R(0)\} \geq 0$. Then, solution of the SEIQR model in equations (2)-(6) are non-negative in the interval $[0, \infty)^{6}$.

Proof:

Assume that $t_{1}=\sup \{t>0: S>0, E>0, I>0, R>0, Q>0, \in[0, t]\}$. Thus, $t_{1}>0$.

It follows from equation (2) that

$$
\frac{d S(t)}{d t}=\Lambda-\left(\lambda+d_{1}\right) S(t)
$$

where $\lambda=\alpha I$

It can be re-written in the following form ${ }^{3}$

$$
\frac{d}{d t}\left[S(t) \exp \left(d_{1} t+\int_{0}^{t} \lambda(\tau) d \tau\right)\right]=\Lambda \exp \left(d_{1} t+\int_{0}^{t} \lambda(\tau) d \tau\right)
$$

Hence, we have 


$$
S\left(t_{1}\right) \exp \left(d_{1} t_{1}+\int_{0}^{t_{1}} \lambda(\tau) d \tau\right)-S(0)=\int_{0}^{t_{1}} \Lambda \exp \left(d_{1} x+\int_{0}^{x} \lambda(\tau) d \tau\right) d x
$$

For $S(0)>0$, it gives

$$
S\left(t_{1}\right) \exp \left(d_{1} t_{1}+\int_{0}^{t_{1}} \lambda(\tau) d \tau\right) \geq \int_{0}^{t_{1}} \Lambda \exp \left(d_{1} x+\int_{0}^{x} \lambda(\tau) d \tau\right) d x
$$

Thus, we obtain

$$
S\left(t_{1}\right) \geq \exp \left(-d_{1} t_{1}-\int_{0}^{t_{1}} \lambda(\tau) d \tau\right) \times \int_{0}^{t_{1}} \Lambda \exp \left(d_{1} x+\int_{0}^{x} \lambda(\tau) d \tau\right) d x>0
$$

Then, we get

$$
S\left(t_{1}\right)>0
$$

In similar manner, it can be shown that $E(t)>0, I(t)>0, Q(t)>0$, and $R(t)>0^{3}$.

\subsection{Theorem 2 (the domain of solutions)}

All the solutions of the model structure that initiate in $\mathbb{R}_{+}^{5}$ are bounded inside the region $\psi$ defined by $\psi=\left\{(S, E, I, Q, R) \in \mathbb{R}^{5}: 0 \leq N(t) \leq \frac{\Lambda}{d_{1}}\right\}_{t \rightarrow \infty} \quad{ }^{6}$

Proof:

By differentiating both sides of equation (1), we obtain

$$
N^{\prime}(t)=S^{\prime}(t)+E^{\prime}(t)+I^{\prime}(t)+R^{\prime}(t)+Q^{\prime}(t)
$$

Substituting from the model (2)-(6), we obtain

$$
N^{\prime}(t)=\Lambda-d_{1} N(t)-d_{2}(Q(t)+I(t))
$$

From theorem 1 , we have $d_{2}(Q(t)+I(t)) \geq 0$; hence, the following inequality is valid:

$$
N^{\prime}(t)+d_{1} N(t) \leq \Lambda
$$

Then, we obtain 


$$
N(t) \leq\left(N(0)-\frac{\Lambda}{d_{1}}\right) e^{-d_{1} t}+\frac{\Lambda}{d_{1}}
$$

Then, when $t \rightarrow \infty$ we obtain the solution

$$
0 \leq N(t) \leq \frac{\Lambda}{d_{1}}
$$

which completes the proof $3,6,25$.

2.4 The equilibrium of the SEIQR model

To determine the equilibrium of this model, we set all the derivatives equal to zero and solve the system as follows ${ }^{3,6,25}$ :

$$
S^{\prime}(t)=E^{\prime}(t)=I^{\prime}(t)=Q^{\prime}(t)=R^{\prime}(t)=0 \rightarrow\{S, E, I, R, Q\} \equiv \text { constants }
$$

which gives

$$
\begin{gathered}
0=\Lambda-\alpha S I-d_{1} S \\
0=\alpha S I-\varepsilon_{1} E \\
0=r E-\varepsilon_{2} I \\
0=\sigma_{1} Q+\sigma_{2} I+\sigma_{3} E-d_{1} R \\
0=\beta_{1} E+\beta_{2} I-\varepsilon_{3} Q
\end{gathered}
$$

From equation (21), we have

$$
I=\frac{r}{\varepsilon_{2}} E
$$

Substituting equation (24) into equation (20) for $E \neq 0$, we obtain

$$
S=\frac{\varepsilon_{1} \varepsilon_{2}}{\alpha r}
$$

Substituting equations (24) and (25) into equation (19), we obtain

$$
E=\frac{\varepsilon_{2} d_{1}}{r \alpha}\left(\Re_{0}-1\right)
$$

where 


$$
\mathfrak{R}_{0}=\frac{\alpha r \Lambda}{d_{1} \varepsilon_{1} \varepsilon_{2}}
$$

Substituting equation (26) into equation (24), we obtain

$$
I=\frac{d_{1}}{\alpha}\left(\mathfrak{R}_{0}-1\right)
$$

Substituting equations (26) and(28) into equation (23), we obtain

$$
Q=\frac{\left(\beta_{1} \varepsilon_{2}+\beta_{2} r\right) d_{1}}{\alpha r}\left(\Re_{0}-1\right)
$$

Substituting equations (26), (28), and (29) into equation (22), we obtain

$$
R=\left(\frac{\sigma_{1}\left(\beta_{1} \varepsilon_{2}+\beta_{2} r\right)+\sigma_{2} r+\varepsilon_{2} \sigma_{3}}{\alpha r}\right)\left(\mathfrak{R}_{0}-1\right)
$$

We can see that at disease-free equilibrium (DFE) $\mathfrak{R}_{0}=1$ i.e. $\frac{\varepsilon_{1} \varepsilon_{2}}{r \alpha}=\frac{\Lambda}{d_{1}}$, which leads to $E=I=Q=R=0$, as in equations (19) and (25), which agrees with the domain of solution in (17).

The number $\mathfrak{R}_{0}$ is called the reproduction number (RBN), which takes the form ${ }^{3,6,25}$ :

$$
\mathfrak{R}_{0}=\frac{\alpha r \Lambda}{d_{1} \varepsilon_{1} \varepsilon_{2}}=\frac{\alpha r \Lambda}{d_{1}\left(r+\beta_{1}+\sigma_{3}+d_{1}\right)\left(\beta_{2}+\sigma_{2}+d_{1}+d_{2}\right)}
$$

Then, if $\mathfrak{R}_{0}>1$ the system has a unique endemic equilibrium ${ }^{8}$ :

$$
E_{0}^{*}=\left(S^{*}, E^{*}, I^{*}, Q^{*}, R^{*}\right)
$$

where

$$
\begin{aligned}
& S^{*}=\frac{\varepsilon_{1} \varepsilon_{2}}{r \alpha}, E^{*}=\frac{\varepsilon_{2} d_{1}}{r \alpha}\left(\Re_{0}-1\right), I^{*}=\frac{d_{1}}{\alpha}\left(\Re_{0}-1\right), Q^{*}=\frac{\left(\beta_{1} \varepsilon_{2}+\beta_{2} r\right) d_{1}}{\alpha r}\left(\Re_{0}-1\right), \text { and } \\
& R^{*}=\left(\frac{\sigma_{1}\left(\beta_{1} \varepsilon_{2}+\beta_{2} r\right)+\sigma_{2} r+\varepsilon_{2} \sigma_{3}}{\alpha r}\right)\left(\mathfrak{R}_{0}-1\right) .
\end{aligned}
$$


Thus, the system has a unique disease-free equilibrium $E_{0}=\left(\frac{\Lambda}{d_{1}}, 0,0,0,0\right)$ when $\Re_{0}=1$, and has a unique endemic equilibrium $E_{0}^{*}=\left(S^{*}, E^{*}, I^{*}, Q^{*}, R^{*}\right)$ when $\Re_{0}>1^{6,8}$.

When $\mathfrak{R}_{0}=0$ there is no transmission, where $\alpha=0.0$, it can be interpreted as the number of secondary cases or the new infection rate (transmission rate at which the susceptible individual converted to an exposed individual) ${ }^{6,8}$.

2.5 Reproduction number by using the Jacobian matrix

To obtain the reproduction number $\mathfrak{R}_{0}$ by using the Jacobian matrix method, we consider that the disease-free equilibrium (DFE) of the SEIQR model is acquired by setting $E=I=R=Q=0$ in equations (19)-(23). Hence, we obtain DFE in form $E_{0}=\left(\frac{\Lambda}{d_{1}}, 0,0,0,0\right) 6$.

The Jacobian matrix of the SEIQR model takes the following form:

$$
J=\left[\begin{array}{ccccc}
-\alpha I-d_{1} & 0 & -\alpha S & 0 & 0 \\
\alpha I & -\varepsilon_{1} & \alpha S & 0 & 0 \\
0 & r & -\varepsilon_{2} & 0 & 0 \\
0 & \sigma_{3} & \sigma_{2} & -d_{1} & \sigma_{1} \\
0 & \beta_{1} & \beta_{2} & 0 & -\varepsilon_{3}
\end{array}\right]
$$

First, we will linearize the first two equations by using the Jacobian method. The first two equations have a disease-free equilibrium (DFE) situation when $I=0 \rightarrow E=0$ and $S=\frac{\Lambda}{d_{1}}$.

Hence, we consider $S^{\prime}(t)=F(S, I)$ and $E^{\prime}(t)+\varepsilon_{1} E(t)=G(S, I)$ such that ${ }^{6}$ :

$$
\begin{gathered}
F(S, I)=\Lambda-\alpha S(t) I(t)-d_{1} S(t) \\
G(S, I)=\alpha S(t) I(t)
\end{gathered}
$$

Then, we have 


$$
\begin{aligned}
{\left[\begin{array}{c}
S^{\prime}(t) \\
E^{\prime}(t)+\varepsilon_{1} E(t)
\end{array}\right] } & =\left[\begin{array}{ll}
\frac{\partial F}{\partial S} & \frac{\partial F}{\partial I} \\
\frac{\partial G}{\partial S} & \frac{\partial G}{\partial I}
\end{array}\right]_{t=0}\left[\begin{array}{c}
S(t)-S(0) \\
I(t)-I(0)
\end{array}\right] \\
& =\left[\begin{array}{cc}
-\alpha I(0)-d_{1} & -\alpha S(0) \\
\alpha I(0) & \alpha S(0)
\end{array}\right]\left[\begin{array}{c}
S(t)-S(0) \\
I(t)-I(0)
\end{array}\right]
\end{aligned}
$$

By substituting from the equilibrium position, we obtain

$$
\left[\begin{array}{c}
S^{\prime}(t) \\
E^{\prime}(t)+\varepsilon_{1} E(t)
\end{array}\right]=\left[\begin{array}{cc}
-d_{1} & -\frac{\alpha \Lambda}{d_{1}} \\
0 & \frac{\alpha \Lambda}{d_{1}}
\end{array}\right]\left[\begin{array}{c}
S(t)-\frac{\Lambda}{d_{1}} \\
I(t)
\end{array}\right]
$$

Hence, the system of nonlinear equations (2) and (3) has been converted to the following linear system ${ }^{6}$ :

$$
\frac{d S(t)}{d t}=\Lambda-d_{1} S(t)-\frac{\alpha \Lambda}{d_{1}} I(t)
$$

and

$$
\frac{d E(t)}{d t}=\frac{\alpha \Lambda}{d_{1}} I(t)-\varepsilon_{1} E(t)
$$

For the complete system at equilibrium, the stability of the disease-free equilibrium (DFE) is given by the Jacobian matrix:

$$
J_{E_{0}}=\left[\begin{array}{ccccc}
-d_{1} & 0 & -\frac{\alpha \Lambda}{d_{1}} & 0 & 0 \\
0 & -\varepsilon_{1} & \frac{\alpha \Lambda}{d_{1}} & 0 & 0 \\
0 & r & -\varepsilon_{2} & 0 & 0 \\
0 & \sigma_{3} & \sigma_{2} & -d_{1} & \sigma_{1} \\
0 & \beta_{1} & \beta_{2} & 0 & -\varepsilon_{3}
\end{array}\right]
$$

By calculating the characteristic equation given by $\left|J_{E_{0}}-\lambda I_{5}\right|=0$, where $\lambda$ is the eigenvalue parameter and $I_{5}$ is the identity matrix of order 5 , then the eigenvalues of the matrix $J_{E_{0}}$ take the following values: 


$$
\left[\begin{array}{l}
\lambda_{1} \\
\lambda_{2} \\
\lambda_{3}
\end{array}\right]=\left[\begin{array}{l}
-\varepsilon_{3} \\
-d_{1} \\
-d_{1}
\end{array}\right]
$$

and the remaining roots are the solution to the following equation:

$$
\left|\begin{array}{cc}
-\varepsilon_{1}-\lambda & \frac{\alpha \Lambda}{d_{1}} \\
r & -\varepsilon_{2}-\lambda
\end{array}\right|=0
$$

which gives

$$
\left(\varepsilon_{1}+\lambda\right)\left(\varepsilon_{2}+\lambda\right)-\frac{r \alpha \Lambda}{d_{1}}=0
$$

The roots of the above equation after inserting $\mathfrak{R}_{0}$ will take the forms:

$$
\lambda_{4}=-\frac{1}{2}\left[\left(\varepsilon_{1}+\varepsilon_{2}\right)-\sqrt{\left(\varepsilon_{1}-\varepsilon_{2}\right)^{2}+4 \varepsilon_{1} \varepsilon_{2} \Re_{0}}\right], \lambda_{5}=-\frac{1}{2}\left[\left(\varepsilon_{1}+\varepsilon_{2}\right)+\sqrt{\left(\varepsilon_{1}-\varepsilon_{2}\right)^{2}+4 \varepsilon_{1} \varepsilon_{2} \Re_{0}}\right]
$$

The formulas (44) generate the following cases ${ }^{6}$ :

1- If $\mathfrak{R}_{0}<1$, then we have $\lambda_{4}<0$ and $\lambda_{5}<0$ in which the disease-free equilibrium $E_{0}$ is locally asymptotically stable.

2- If $\mathfrak{R}_{0}>1$, then we have $\lambda_{4}>0$ and $\lambda_{5}<0$ in which the endemic equilibrium $E_{0}^{*}$ is locally asymptotically unstable.

3- If $\mathfrak{R}_{0}=1$, then we have $\lambda_{4}=0$ and $\lambda_{5}<0$ in which the disease-free equilibrium $E_{0}$ is locally asymptotically unstable.

\subsection{Condition of equilibrium (Hartman-Grobman theorem)}

The Hartman-Grobman theorem states that the solutions of a square system of nonlinear ordinary differential equations (2)-(5) in a neighbourhood of a steady-state look "qualitatively" similar to the solutions of the linearized system near the point $E_{0}=\left(\frac{\Lambda}{d_{1}}, 0,0,0,0\right)$. This result 
holds only when the equilibrium is hyperbolic, that is, when none of the eigenvalues of the matrix $J_{E_{0}}$ have zero real part ${ }^{6}$.

Thus, from (41), we obtain the following condition of equilibrium:

$$
\alpha r \Lambda-d_{1} \varepsilon_{1} \varepsilon_{2} \neq 0
$$

\subsection{The uniqueness of equilibrium condition}

If the matrix $J_{E_{0}}$ is obtained from the linearization and is the Jacobian evaluated at equilibrium $\operatorname{DFE}\left(E_{0}\right)=\left(\frac{\Lambda}{d_{1}}, 0,0,0,0\right)$, the condition $\left|J_{E_{0}}\right| \neq 0$ means that the equilibrium is isolated, which means there is a disk around it that does not contain other equilibria $6,18,23,25$.

Hence, from (40), we have

$$
\left|J_{E_{0}}\right|=\left|\begin{array}{ccccc}
-d_{1} & 0 & -\frac{\alpha \Lambda}{d_{1}} & 0 & 0 \\
0 & -\varepsilon_{1} & \frac{\alpha \Lambda}{d_{1}} & 0 & 0 \\
0 & r & -\varepsilon_{2} & 0 & 0 \\
0 & \sigma_{3} & \sigma_{2} & -d_{1} & \sigma_{1} \\
0 & \beta_{1} & \beta_{2} & 0 & -\varepsilon_{3}
\end{array}\right|
$$

which gives

$$
\left|J_{E_{0}}\right|=\varepsilon_{3} d_{1}\left(\alpha r \Lambda-d_{1} \varepsilon_{1} \varepsilon_{2}\right)=\frac{\varepsilon_{3} d_{1}}{\varepsilon_{1} \varepsilon_{2}}\left(\frac{\alpha r \Lambda}{d_{1} \varepsilon_{1} \varepsilon_{2}}-1\right) \neq 0
$$

Thus, the condition (45) is the only condition of the equilibrium of the SEIQR model.

Therefore, the unique equilibrium condition of the SEIQR model is

$$
\frac{\alpha r \Lambda}{d_{1} \varepsilon_{1} \varepsilon_{2}}-1=\mathfrak{R}_{0}-1 \neq 0
$$

The reproduction number $(\mathrm{RBN}) \mathfrak{R}_{0}=\frac{\alpha r \Lambda}{d_{1} \varepsilon_{1} \varepsilon_{2}}$ is also unique ${ }^{6}$.

2.8 Local sensitivity analysis of $\operatorname{RBN}\left(\Re_{0}\right)$ 
Local sensitivity analysis is a sensitivity analysis that examines the change in the output values that results from a change in one input value (parameter) ${ }^{6}$.

The sensitivity or elasticity of quantity $G$ concerning parameter $\mathrm{p}$ is given by:

$$
\wp_{G}^{p}=\frac{\partial G}{\partial p} / \frac{G}{p}= \pm \frac{\% \Delta G}{\% \Delta p}
$$

The sensitivity of $G$ concern $p$ is positive if $G$ is increasing concerning $\mathrm{p}$ and negative if $G$ is decreasing concerning $\mathrm{p}$.

Applying formula (49) into reproduction number $\mathfrak{R}_{0}$, which takes the form ${ }^{6}$ :

$$
\mathfrak{R}_{0}=\frac{\alpha r \Lambda}{d_{1} \varepsilon_{1} \varepsilon_{2}}=\frac{\alpha r \Lambda}{d_{1}\left(r+\beta_{1}+\sigma_{3}+d_{1}\right)\left(\beta_{2}+\sigma_{2}+d_{1}+d_{2}\right)}
$$

Then,

$$
\begin{gathered}
\wp_{\mathfrak{R}_{0}}^{\alpha}=\frac{\partial \mathfrak{R}_{0}}{\partial \alpha} /\left(\frac{\mathfrak{R}_{0}}{\alpha}\right)=1>0 \\
\wp_{\mathfrak{R}_{0}}^{r}=\frac{\partial \mathfrak{R}_{0}}{\partial r} /\left(\frac{\mathfrak{R}_{0}}{r}\right)=1-\frac{r}{\varepsilon_{1}}>0 \\
\wp_{\mathfrak{R}_{0}}^{d_{1}}=\frac{\partial \mathfrak{R}_{0}}{\partial d_{1}} /\left(\frac{\mathfrak{R}_{0}}{d_{1}}\right)=-\left(\frac{d_{1}\left(\varepsilon_{1}+\varepsilon_{2}\right)}{\varepsilon_{1} \varepsilon_{2}}+1\right)<0 \\
\wp_{\mathfrak{\Re}_{0}}^{\beta_{1}}=\frac{\partial \mathfrak{R}_{0}}{\partial \beta_{1}} /\left(\frac{\mathfrak{R}_{0}}{\beta_{1}}\right)=-\frac{\beta_{1}}{\varepsilon_{1}}<0 \\
\wp_{\mathfrak{R}_{0}}^{\sigma_{3}}=\frac{\partial \mathfrak{R}_{0}}{\partial \sigma_{3}} /\left(\frac{\mathfrak{R}_{0}}{\sigma_{3}}\right)=-\frac{\sigma_{3}}{\varepsilon_{1}}<0 \\
\wp_{\mathfrak{R}_{0}}^{d_{2}}=\frac{\partial \mathfrak{R}_{0}}{\partial d_{2}} /\left(\frac{\mathfrak{R}_{0}}{d_{2}}\right)=-\frac{d_{2}}{\varepsilon_{2}}<0 \\
\wp_{\mathfrak{R}_{0}}^{\beta_{2}}=\frac{\partial \mathfrak{R}_{0}}{\partial \beta_{2}} /\left(\frac{\mathfrak{R}_{0}}{\beta_{2}}\right)=-\frac{\beta_{2}}{\varepsilon_{2}}<0 \\
\wp_{\mathfrak{R}_{0}}^{\sigma_{2}}=\frac{\partial \mathfrak{R}_{0}}{\partial \sigma_{2}} /\left(\frac{\mathfrak{R}_{0}}{\sigma_{2}}\right)=-\frac{\sigma_{2}}{\varepsilon_{2}}<0
\end{gathered}
$$


It means that a $1 \%$ increase in each one $\left(d_{1}, \beta_{1}, \sigma_{3}, d_{2}, \sigma_{2}, \beta_{2}\right)$ will produce $\left(\left(\frac{d_{1}\left(\varepsilon_{1}+\varepsilon_{2}\right)}{\varepsilon_{1} \varepsilon_{2}}+1\right), \frac{\beta_{1}}{\varepsilon_{1}}, \frac{\sigma_{3}}{\varepsilon_{1}}, \frac{d_{2}}{\varepsilon_{2}}, \frac{\sigma_{2}}{\varepsilon_{2}}, \frac{\beta_{2}}{\varepsilon_{2}}\right) \%$ a decrease in $\mathfrak{R}_{0}$, respectively, and a $1 \%$ increase in $r$ will produce $\left(1-\frac{r}{\varepsilon_{1}}\right)$ an increase in $\operatorname{RBN}\left(\Re_{0}\right)$. From relation $(51), \wp_{\mathfrak{R}_{0}}^{\alpha}=1$ means that a $1 \%$ increase $\alpha$ will produce a rise of $1 \%$ in $\mathfrak{R}_{0}{ }^{6}$.

2.9 Global stability of equilibria of the SEIQR model (Lyapunov stability theorem)

One of the most commonly used functions is the Lyapunov function. Lyapunov functions are scalar functions that may be used to prove the global stability of equilibrium. Lyapunov states that if a function $V(x)$ is globally positively definite and radially unbounded and its time derivative is globally negative, $V^{\prime}(x)<0$ for all $x \neq x^{*}$ then the equilibrium $x^{*}$ is globally stable for the autonomous system $x^{\prime}=f(x)$, and $V(x)$ is called a Lyapunov function ${ }^{6}$.

\subsection{Theorem 5 (global stability)}

The SEIQR model $\operatorname{DFE}\left(E_{0}\right)=\left(\frac{\Lambda}{\varepsilon_{1}}, 0,0,0,0\right)$ is globally stable in disease-free equilibrium under the condition $\mathfrak{R}_{0}<1$.

Proof:

We will consider the SEIQR model on the space of the first three variables only $(S, E, I)$. It is clear that if the disease-free equilibrium for the first three equations is globally stable, then $(R, Q) \rightarrow 0$ and the disease-free equilibrium for the full SEIQR model is globally stable.

We construct the Lyapunov function on $\mathbb{R}_{+}^{3}$ in the following form:

$$
V=\kappa\left(S-S^{*}-S^{*} \ln \left(\frac{S}{S^{*}}\right)\right)+\frac{E}{\varepsilon_{1}}+\frac{I}{r}
$$


where $\kappa$ is a parameter that will be determined later, and $S^{*}=\frac{\Lambda}{d_{1}}$.

The equation (59) shows that at the disease-free equilibrium $\left(S^{*}=\frac{\Lambda}{d_{1}}, 0,0\right), V=0$.

Now, we have to show that $V>0$ for all $(S, E, I) \geq\left(\frac{\Lambda}{d_{1}}, 0,0\right)$.

The equation (59) can be rewritten as follows:

$$
V=\kappa S^{*}\left(\frac{S}{S^{*}}-1-\ln \left(\frac{S}{S^{*}}\right)\right)+\frac{E}{\varepsilon_{1}}+\frac{I}{r}
$$

The first term is positive for any value of $S / S^{*}$, and the other two terms are also non-negative, so $V>0$

Now, we take the derivative of equation (59); we obtain

$$
V^{\prime}=\kappa\left(1-\frac{S^{*}}{S}\right) S^{\prime}+\frac{E^{\prime}}{\varepsilon_{1}}+\frac{I^{\prime}}{r}
$$

Substituting from the first three equations of the SEIQR model and using the equation (25), we obtain

$$
V^{\prime}=2 \Lambda \kappa-\frac{\kappa \alpha \varepsilon_{3}}{r} S I-d_{1} \kappa S-\frac{\Lambda^{2} \kappa}{S d_{1}}+\frac{\kappa \alpha \Lambda \varepsilon_{3}}{d_{1} r} I+\frac{\alpha \varepsilon_{3}}{\varepsilon_{1} r} S I-\frac{\varepsilon_{3}}{r} I
$$

We choose $\kappa=\frac{1}{\varepsilon_{1}}$, then we have

$$
V^{\prime}=-\frac{\Lambda}{\varepsilon_{1}}\left(\frac{\Lambda}{d_{1} S}+\frac{d_{1} S}{\Lambda}-2\right)+\frac{\varepsilon_{2}}{r}\left(\Re_{0}-1\right) I
$$

Since $\mathfrak{R}_{0}<1$ then, the last term is non-positive.

For the first term, consider $\frac{d_{1} S}{\Lambda}=x$; then, the term inside the brackets takes the form 


$$
\left(x+\frac{1}{x}-2\right)=\frac{(x-1)^{2}}{x}>0 \text {, and now we have two possibilities. The first term is at the }
$$

equilibrium point, where we have $S=S^{*}=\frac{\Lambda}{d_{1}}$, and it gives $x=1$. Then, the first term completely vanishes, and then we have the last term only, which is already non-negative. Thus, $V^{\prime}<0$.

The second possibility is $x \neq 1$; then, the two terms are non-positive. Thus, $V^{\prime}<0$.

Hence, we have $V^{\prime}<0$ for every $(S(t), E(t), I(t)) \geq\left(\frac{\Lambda}{d_{1}}, 0,0\right)^{6}$.

Therefore, by the Lyapunov theorem, the disease-free equilibrium is globally asymptotically stable for the system of the SEIQR model in all.

2.11 Solutions for the system of the SEIQR model

We assume the initial conditions of the SEIQR system in (38), (39), and (4)-(6) to take the form

$$
\left.\{S(t), E(t), I(t), R(t), Q(t)\}\right|_{t=0}=\{S(0), E(0), I(0), R(0), Q(0)\}
$$

We solved this system by using MAPLE software. Hence, we obtain

$$
I(t)=\frac{1}{\alpha \Lambda \delta}\left[\left(\left(-\varepsilon_{1}+\varepsilon_{2}\right)-\delta\right) \gamma_{1} e^{\frac{\delta}{2 d_{1}} t}+\left(\left(\varepsilon_{1}-\varepsilon_{2}\right)-\delta\right) \gamma_{2} e^{-\frac{\delta}{2 d_{1}} t}\right] e^{-\frac{1}{2}\left(\varepsilon_{1}+\varepsilon_{2}\right) t}
$$

where $\delta=\sqrt{4 \Lambda \alpha r d_{1}+d_{1}^{2} \varepsilon_{1}^{2}-2 d_{1}^{2} \varepsilon_{1} \varepsilon_{2}+d_{1}^{2} \varepsilon_{2}^{2}}, \gamma_{1}=\left(d_{1}\left(\varepsilon_{1}-\varepsilon_{2}\right)-\delta\right) E(0)+2 \Lambda \alpha I(0)$, and $\gamma_{2}=\left(d_{1}\left(\varepsilon_{1}-\varepsilon_{2}\right)+\delta\right) E(0)+2 \Lambda \alpha I(0)$

Consequently, we can obtain the other functions $S, E, I$, and $Q$.

2.12 Model verification and predictions

To verify the SEIQR model, we will apply it to the real data regarding the COVID-19 outbreak in Saudi Arabia. COVID-19 has been in Saudi Arabia since March 3, 2020. Cases continued to be discovered in small numbers until the beginning of April, and then the number 
of detected cases increased. Therefore, we decided in this study to consider April 1, 2020, as the real beginning of the spread of the COVID-19 epidemic in Saudi Arabia.

We used tables of statistics issued from the Saudi Ministry of Health ${ }^{33}$ and the daily official statement issued by the ministry as well as Wikipedia ${ }^{34}$, which also depends on the ministry's website and other websites that would announce these statistics.

Another source of these data is the "Saudi Centre for Disease Prevention and Control ${ }^{35}$." We used the official website of the General Statistics Authority of Saudi Arabia for more information about the kingdom's population, mortality rate, and population growth rate.

To study the spread of COVID-19 in Saudi Arabia before June 13, 2020, we will represent the curve of the number of daily infections and the time series curve of the total number of infections, as shown in Figs. 2 and 3:

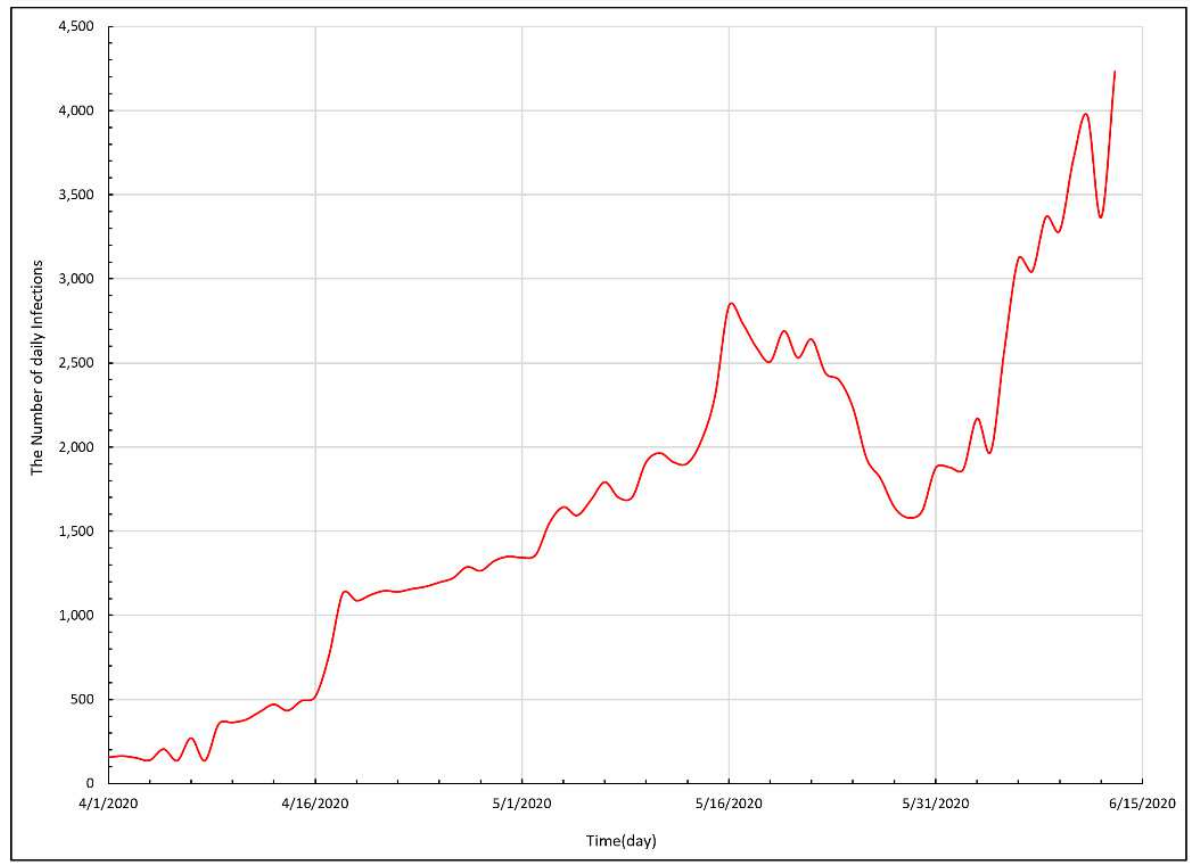

Figure 2. The real number of daily infections in Saudi Arabia between 4/1/2020 and $6 / 13 / 2020$ 
Fig. 2 shows that the number of cases on April 1, 2020, was 157 infections, and it reached 4233 infections on June 13, 2020. Between the two numbers, the curves passed through many up and down variations.

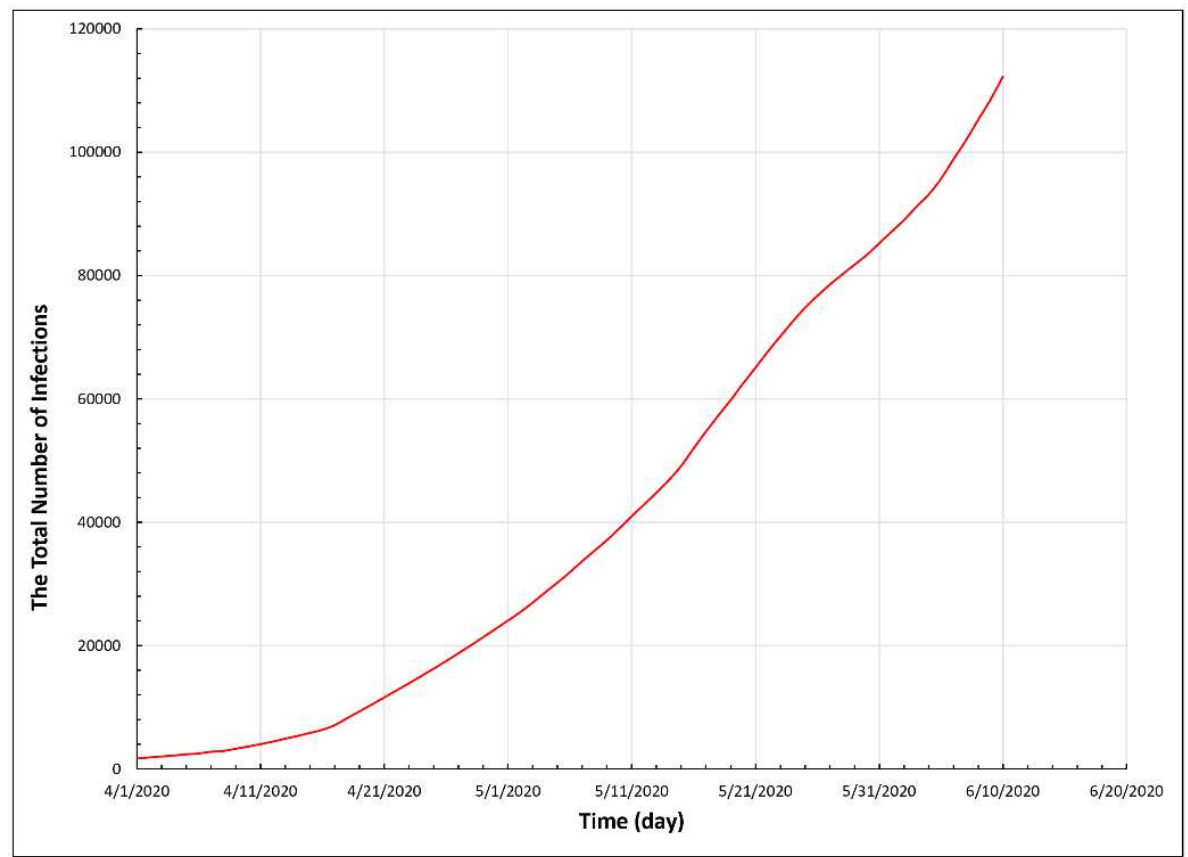

Figure 3. The total number of infections in Saudi Arabia between 4/1/2020 and 6/10/2020

Fig. 3 shows that the total number of cases at the same interval started with 157 infections and reached an accumulated amount of 122,259 infections on June 13, 2020. Therefore, we will use these data through the present SEIQR model to discern whether there is a convergence between the model results and the real data ${ }^{33-35}$.

\section{Results}

3.1 Applying the SEIQR model to Saudi Arabia data of the spread of Covid-19

According to the official data of Saudi Arabia, we have the following initial data, which are considered the initial conditions of the system based on the SEIQR model, as in Table $1^{33-35}$ : 
Table 1: The initial conditions of the SEIQR model

\begin{tabular}{lllll}
\hline$S(0)$ & $E(0)$ Assumed & $I(0)$ & $R(0)$ & $Q(0)$ \\
\hline $34,218,169$ & $5.0 \times 10^{3}$ & 157 & 99 & 1720 \\
\hline
\end{tabular}

where $S(0)=34,218,169$ is the total population in Saudi Arabia up to June 13, 2020. The total number of exposed populations infected but not detected by testing has been assumed $E(0)=5000$, while the number of infections $I(0)=157$. The recovery number of the population at the same time was $R(0)=99$, and the population number in quarantine is $Q(0)=1720$. The total number of new births of Saudi children and new residents $\Lambda \approx 2300$ person/day and the rate of natural deaths is approximately 1030 people/day, which results in $d_{1} \approx 3 \times 10^{-5}$. Some of the other parameters have been calculated, estimated, or assumed, as in Table 2. The estimated data has been calculated by using the most powerful methods, however, is calibration or curve fitting. Curve fitting is the process of identifying the parameters of a curve, or mathematical function, that has the best fit to a series of data points. MAPLE software has been used for the fitting curves and to estimate the parameters we need.

Table 2: The values of parameters in SEIQR 3,8,10,16,20,22,25,32

\begin{tabular}{ccc}
\hline Parameter & Value & Background \\
\hline$\beta_{1}$ & 0.02 & Assumed \\
$\beta_{2}$ & 0.005 & Assumed \\
$\sigma_{1}$ & 0.001 & Calculated \\
$\sigma_{2}$ & 0.002 & Estimated \\
$\sigma_{3}$ & 0.002 & Estimated \\
$r$ & 0.01 & Estimated
\end{tabular}




\section{$d_{1} \quad 3.0 \times 10^{-5} \quad$ Calculated \\ $d_{2} \quad 3.5 \times 10^{-7} \quad$ Calculated}

After using the above values of the parameter and by using MAPLE software, we obtain the results that indicate the number of daily infections as outcomes of the SEIQR model. The following figure shows the numerical results of the SEIQR model against the real data with different values of the parameter $\alpha$ and $\mathrm{RBN}\left(\mathfrak{R}_{0}\right)$ to show the convergence between them. It is noted that an increase in the parameter $\alpha$ leads to a rise in the number of infections and RBN $\left(\mathfrak{R}_{0}\right)$. The value of the parameter $\alpha$ (the rate of transmission from the susceptible population to infection in Saudi Arabia) within the abovementioned interval is $\alpha=(2.0,2.2,2.4) \times 10^{-9}$. Moreover, the reproduction number $\operatorname{RBN}\left(\mathfrak{R}_{0}\right)$ is $\mathfrak{R}_{0}=(6.81,7.49,8.17)>1$. In other words, the transmission rate at which the susceptible individual converted to an exposed individual is higher than one, which means the spread of COVID-19 is unstable.

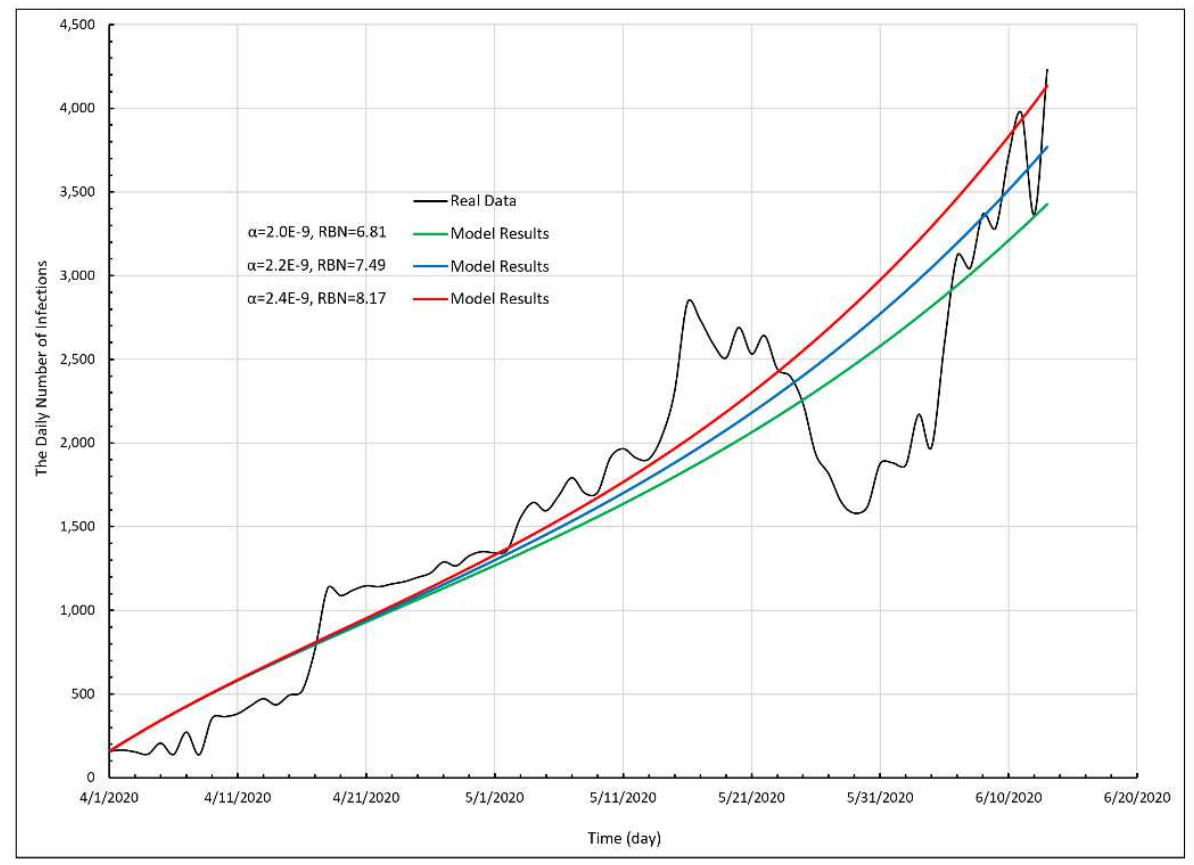

Figure 4. The number of daily infections based on the SEIQR model against the real data in Saudi Arabia between 4/1/2020 and 6/13/2020 
Fig. 4 shows the number of daily infections based on the SEIQR model against the real data in Saudi Arabia between 4/1/2020 and 6/13/2020 with three different values of the rate of transmission from susceptible populations to infection in Saudi Arabia, $\alpha=(2.0,2.2,2.4) \times 10^{-9}$ which gives three different values of $\operatorname{RBN} \Re_{0}=(6.81,7.49,8.17)>1$. It is noted that the three curves that come as results from the SEIQR model work as three trends to the curves belong to the real data, which makes the results due to applying the SEIQR model close to the actual data.

To illustrate the convergence between the results of the proposed SEIQR model and the real results, we displayed Fig. 5, which shows the cumulatively infected numbers within the same interval referred to earlier. It is noted that the curve of the real data is set between the three cases of the SEIQR model with the mentioned values of $\alpha$ and $\Re_{0}$ parameters.

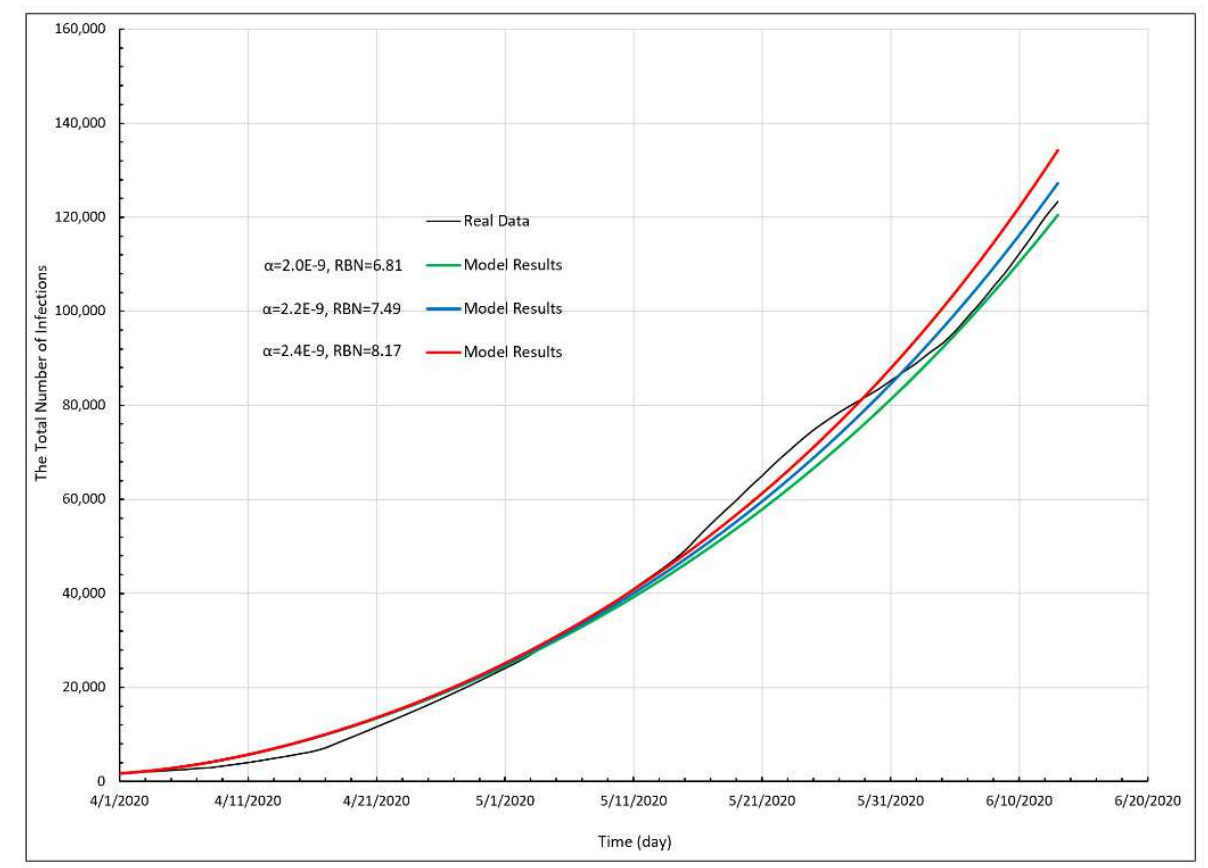

Figure 5. The total number of infections based on the SEIQR model against the real data in Saudi Arabia between 4/1/2020 and 6/13/2020

Now, we will predict the spread of COVID-19 in Saudi Arabia based on the current data and parameters with the same rates without any change in the procedures and considering that 
everything will continue as it is. We will illustrate the results of the total number of infections by applying the SEIQR model for the next three months, starting from April 1, 2020, and ending on October 18, 2020. As shown in Fig. 6, the curves and results show whether the number of infections will be reduced and whether the spread of COVID-19 will continue to be unstable. The curves have been established by using the same three values of the two parameters $\alpha$ and $\mathfrak{R}_{0}$.

The figure shows that the spread of COVID-19 will continue with an unstable situation without being slowed and that the number of daily infections will rise to very high numbers.

Thus, we will later describe the best practices for this situation (best protocol) to control the spread of the COVID-19.

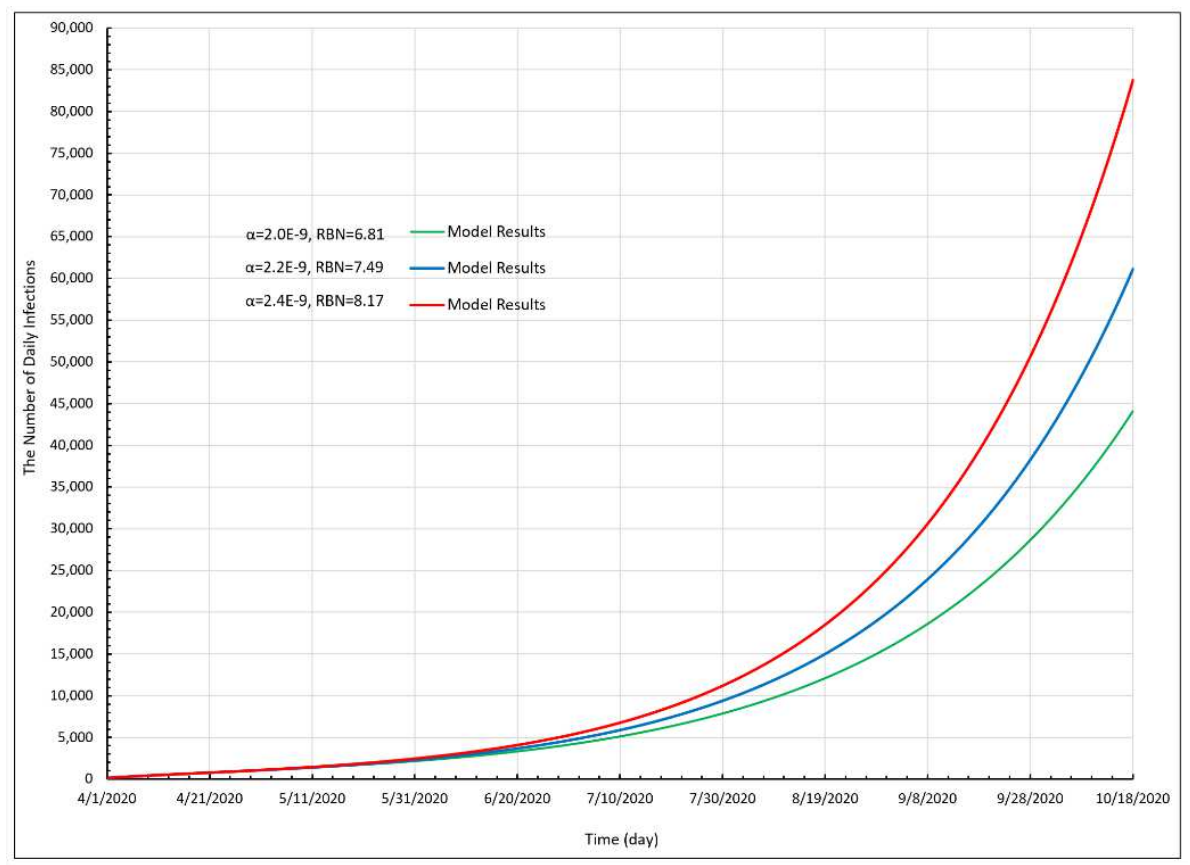

Figure 6. The number of daily infections based on the SEIQR model in Saudi Arabia between $4 / 1 / 2020$ and $10 / 18 / 2020$ 
3.2 Study of the sensitivity of the RBN $\left(\mathfrak{R}_{0}\right)$ based on the current data of Saudi Arabia

To study the sensitivity of the critical parameters against the reproduction number $\left(\mathfrak{R}_{0}\right)$, we use the equations (51)-(58) and represent Fig. 7, which shows the increment of the value of $\operatorname{RBN}\left(\Re_{0}\right)$ concerning the parameters $\alpha, r, \beta_{1}, \sigma_{3}, \sigma_{2}$, and $\beta_{2}$, respectively. For the parameters $d_{1}$ and $d_{2}$, we do not need to study its effects where we cannot change its values. Therefore, we will use the values of that parameter in this study.

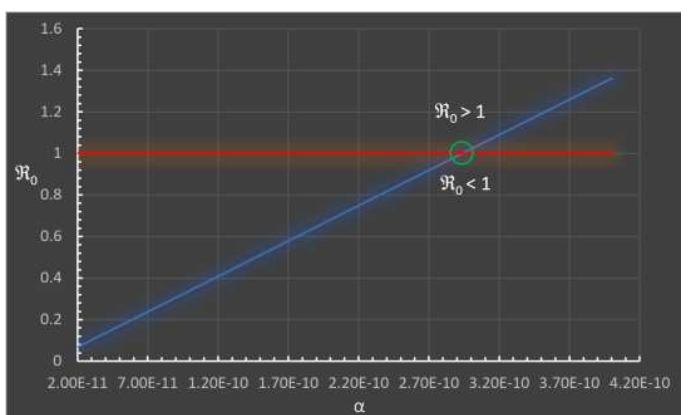

Figure $7 \mathrm{a} . \Re_{0}$ against $\alpha$

$\left(\alpha \leq 2.8 \times 10^{-10} \rightarrow \Re_{0} \leq 1\right)$

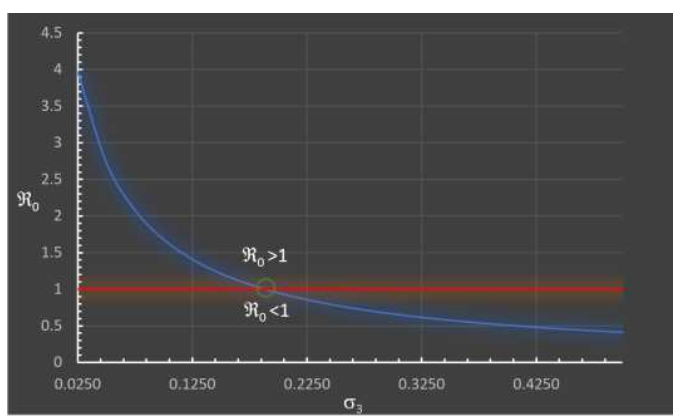

Figure 7c. $\Re_{0}$ against $\sigma_{3}\left(\sigma_{3} \geq 0.18 \rightarrow \Re_{0} \leq 1\right)$

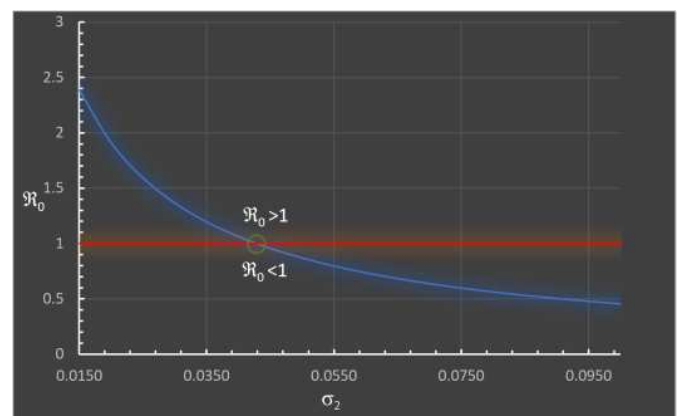

Figure 7e. $\Re_{0}$ against $\sigma_{2}\left(\sigma_{2} \geq 0.04 \rightarrow \Re_{0} \leq 1\right)$

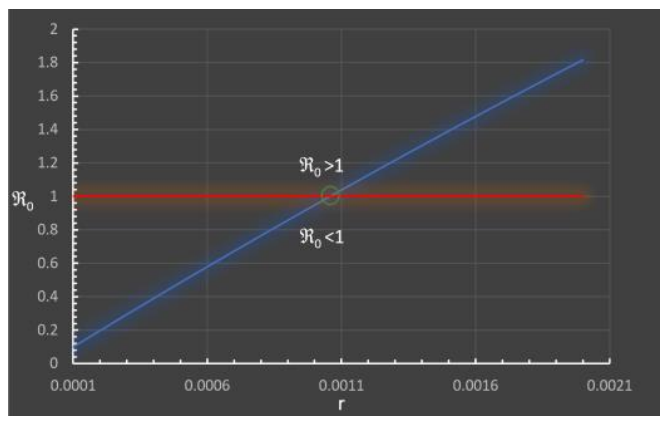

Figure 7b. $\mathfrak{R}_{0}$ against $r\left(r \leq 0.001 \rightarrow \mathfrak{R}_{0} \leq 1\right)$

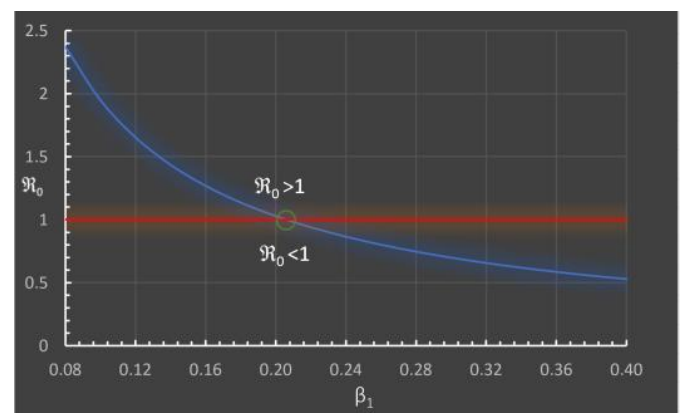

Figure 7 d. $\Re_{0}$ against $\beta_{1}\left(\beta_{1} \geq 0.2 \rightarrow \mathfrak{R}_{0} \leq 1\right)$

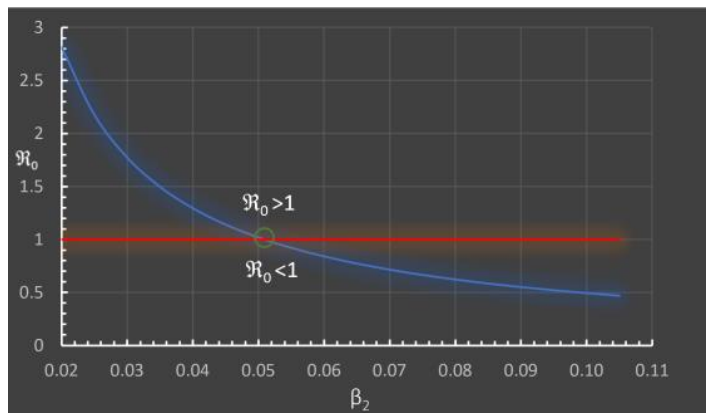

Figure 7f. $\Re_{0}$ against $\beta_{2}\left(\beta_{2} \geq 0.045 \rightarrow \Re_{0} \leq 1\right)$

Figure 7. The sensitivity of the parameters on the value of the $\operatorname{RBN}\left(\Re_{0}\right)$ 
Fig. 7a shows that the parameter $\alpha$ has a significant effect on the value of the reproduction number $\mathfrak{R}_{0}$, where an increase of the parameter $\alpha$ leads to an increase in the value of the reproduction number $\mathfrak{R}_{0}$. The Saudi Arabia data indicate that for a stable epidemic spreading of COVID-19 $\mathfrak{R}_{0}<1$, the value of the parameter $\alpha$ must be smaller than or equal to the value $2.8 \times 10^{-10}$, which is not the situation in the studied time interval.

Fig. $7 \mathrm{~b}-7 \mathrm{f}$ shows that the values of the other parameters have significant effects on the reproduction number $\Re_{0}$. The value of each parameter that gives a stable reproduction number $\left(\mathfrak{R}_{0}<1\right)$ individually, when the other parameters remain constant, is provided as follows:

$$
r \leq 0.001, d_{1}>0.002, \beta_{1}>0.2, \sigma_{3} \geq 0.18, d_{2} \geq 0.4, \sigma_{2} \geq 0.04, \beta_{2} \geq 0.045
$$

The other parameters change within its suitable range, making all its significant private effects, even the value of the reproduction number $\mathfrak{R}_{0}$ higher or smaller than one.

\section{Discussions}

4.1 The current state and how to stop the spread of Covid-19 in Saudi Arabia

Now, we are in the most critical part of the assessment of the current situation and evaluate what needs to take place later in Saudi Arabia to control the COVID-19 spread. Therefore, in this section, we will apply the SEIQR model to analyze the current situation with new initial conditions and different values of the system parameters according to the current state. We will consider June 14, 2020, as the fresh start, and we will renew all the initial conditions in Table I. The number of infections on this day was $I(0)=4223^{33-35}$. We will keep the values of the parameters $d_{1}$ and $d_{2}$ as it is without any change, while the other parameters will take the values in Table 3: 
Table 3: The new values of parameters in SEIQR that give an ideal situation

\begin{tabular}{cll}
\hline Parameter & Value & Background \\
\hline$\beta_{1}$ & 0.2 & Assumed \\
$\beta_{2}$ & 0.01 & Assumed \\
$\sigma_{1}$ & 0.01 & Calculated \\
$\sigma_{2}$ & 0.01 & Estimated \\
$\sigma_{3}$ & 0.02 & Estimated \\
$r$ & 0.003 & Calculated \\
$d_{1}$ & $3.0 \times 10^{-5}$ & Calculated \\
$d_{2}$ & $3.5 \times 10^{-7}$ & Calculated \\
$\alpha$ & $2.0 \times 10^{-10}$ & Assumed
\end{tabular}

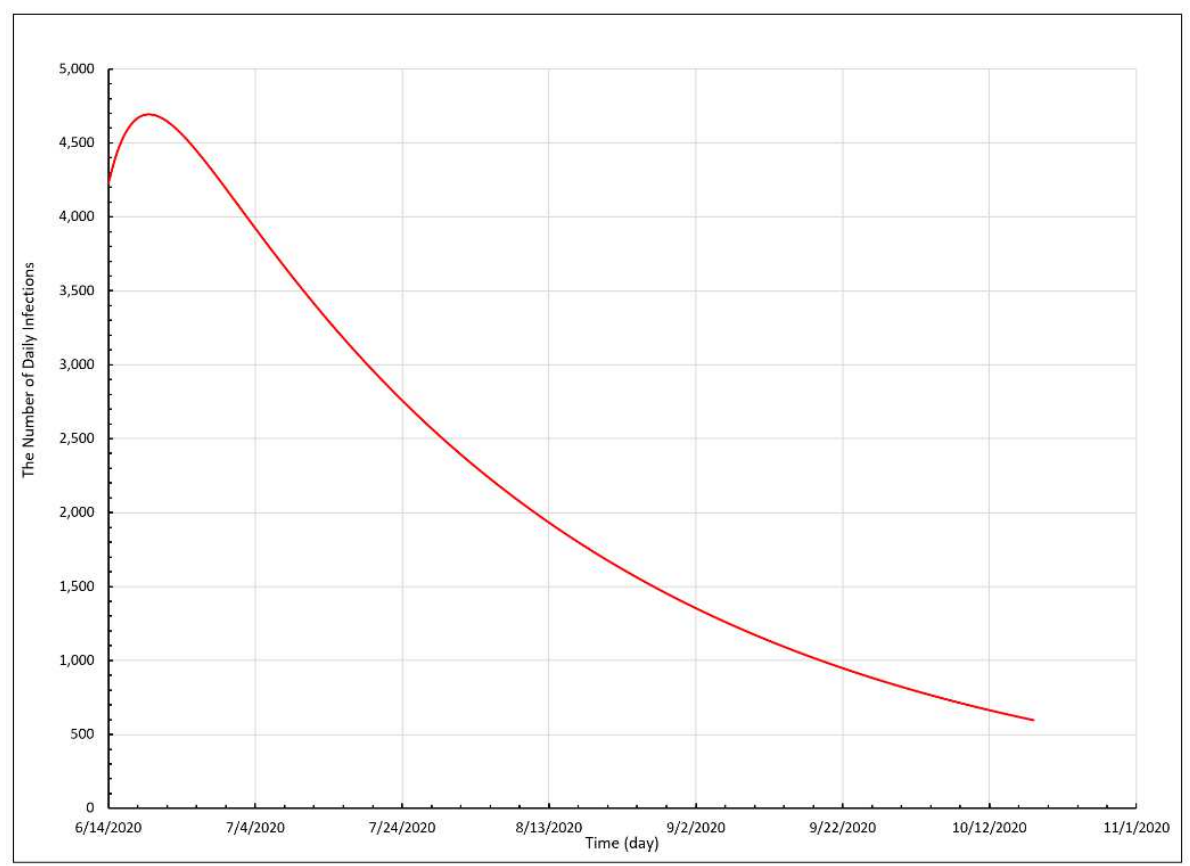

Figure 8. The number of daily infections based on the SEIQR model in Saudi Arabia was between $6 / 14 / 2020$ and 10/18/2020 if the values of the parameters, as in Table 3. 
Figure 8 shows how the current state of spreading COVID-19; moreover, it offers an estimation for the situation in Saudi Arabia up to October 18, 2020.

It is noted in figure 8 that the spreading of COVID-19 in Saudi Arabia passed through its peak point on 16-18 July 2020, which agrees with the actual data; after that, the spread has slowed down and kept this attitude until the current days, and the reproduction number takes the value $\mathfrak{R}_{0}=0.1<1$ which means the situation is stable

According to this curve, we can also see that the number of daily infections on October 18 , 2020, will be approximately 600 persons/day, and we can predict that the spreading situation will go to a more stable position and better state.

4.2 The ideal protocol to halt the spread Covid-19 in Saudi Arabia

To obtain the ideal situation, which can help us break the spread COVID-19 in Saudi Arabia, we must start implementing the following protocols and procedures (see figure 9):

1. Decrease the value of the transmission rate from the susceptible population to infected but not detected by testing the population to be in the following interval $\alpha \leq 2.8 \times 10^{-10}$.

2. Increase the value of the transmission coefficient from an infected population but not detected by testing to a quarantine population $\beta_{1}$ to be $\beta_{1} \geq 0.2$, which means expanding the detection work and the need to isolate infected people in compulsory quarantine areas as an example.

3. Increase the value of the transmission coefficient from the confirmed detected population by testing to a quarantine population $\beta_{2}$ to be $\beta_{2} \geq 0.01$, which means we must help the confirmed infected population, which they need to be in the quarantine zone.

4. Increase the value of the transmission rate from the quarantine population to the recovery zone $\sigma_{1}$ to be $\sigma_{1} \geq 0.001$, which means we must apply a successful treatment on the quarantine area and help them recover. 
5. Increase the value of the transmission rate from the confirmed detected population to the recovery population $\sigma_{2}$ to be $\sigma_{2} \geq 0.01$ by applying a successful treatment for the confirmed infected population and help them recover without needing to go to the quarantine zone.

6. Increasing the value of the transmission rate $\sigma_{3}$ from infected and undetected populations to the recovery zone directly to be $\sigma_{3} \geq 0.02$ by using a successful treatment and supplying with vitamins, health awareness, social spacing, and applying the principle of prevention are better than curing.

7. Increase the value of infected but not detected individuals by checking population to infected population for treatment $r$ to be $r_{1} \geq 0.001$, which means we have to offer the more effective and accurate methods of diagnosis to determine the confirmed infections. Moreover, raising awareness about ways to identify the disease and the symptoms and ways of confirming the infection.

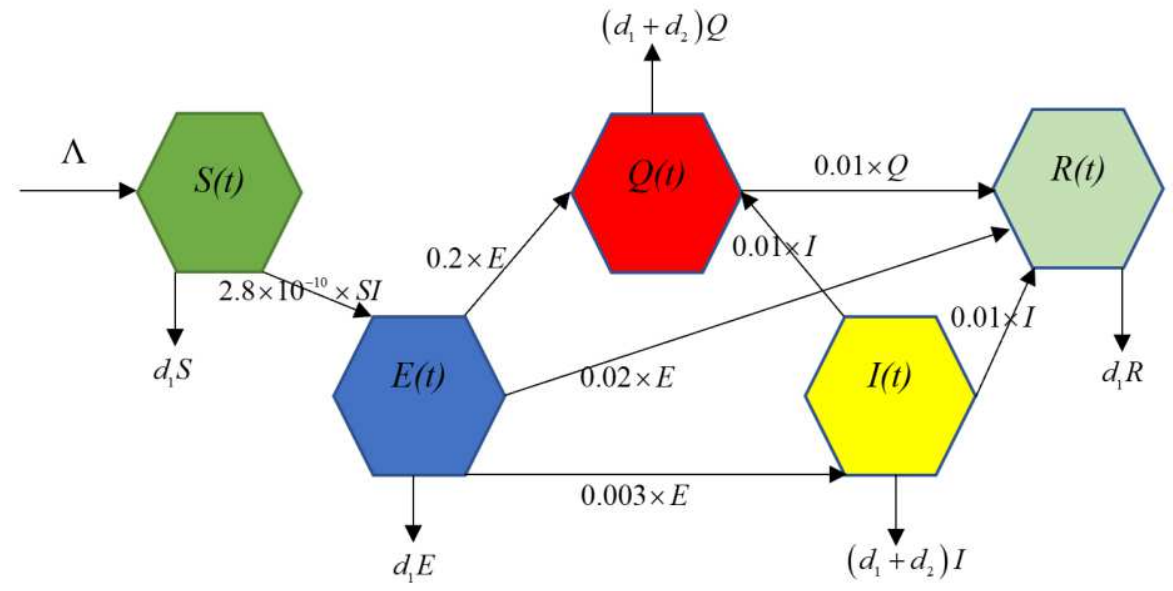

Figure 9. The flowchart of the ideal protocol based on the SEIQR model

\section{Conclusion}

For this analysis, a new statistical outbreak (SEIQR) model was developed for the introduction of the current COVID-19 coronavirus. Throughout the estimation and treatment of 
the COVID-19 outbreak, this pandemic paradigm offers a different method. In Saudi Arabia, the original COVID-19 details were used to validate the effects of the current model. The findings suggest that the SEIQR approach is an excellent tool for the study in Saudi Arabia and other countries of the transmission of diseases, such as COVID-19.

Five measures are included in the optimal procedure, and guidance has been comprehensive in helping delay the spread of COVID-19 in Saudi Arabia. Prevention is safer than recovery, one of the key targets in this procedure.

The main approach to slowing down the transmission of COVID-19 is to remain home and to put sick individuals in a distant location or a protected place as far as possible.

In order to evaluate the reported infections, we need more reliable and effective diagnostic methods. In addition, awareness raising on ways in which the infection can be confirmed, the disease symptoms and ways.

Ultimately, efficient and appropriate care of sick patients must be given, and medicines, tones, and nutrients must be distributed to non-infected individuals to prevent them.

\section{ACKNOWLEDGMENTS}

The authors are very grateful and thank the Research and Development Grants Program for National Research Institutions and Centres (GRANTS), Target Research Program, Infectious Diseases Research Grant Program, King Abdulaziz City for Science and Technology (KACST), and Kingdom of Saudi Arabia for funding this project and this work.

\section{AUTHOR CONTRIBUTIONS}

H.Y., M.E., and N.A. conceived the original idea and led the overall study. A.E. and A.S. wrote the paper and carefully revised the manuscript. H. Y, M. E, N.A., A.E., and A.S. collected and analyzed all data. All the authors read and approved the final manuscript. 


\section{COMPETING INTERESTS}

This work was supported by The Research and Development Grants Program funded this work for National Research Institutions and Centres (GRANTS), the Target Research Program, the Infectious Diseases Research Grant Program, King Abdulaziz City for Science and Technology (KACST), Kingdom of Saudi Arabia [Grant numbers: 5-20-01-007-0002 with amount 270,000.00 SR]. H. Youssef is the P-I of the project, while N. Alghamdi, M. Ezzat, A. El-Bary, and A. Shawky are Co-Is of the project. 


\section{References}

1 Gao, W., Veeresha, P., Prakasha, D., Baskonus, H. M. \& Yel, G. New approach for the model describing the deathly disease in pregnant women using Mittag-Leffler function. Chaos, Solitons \& Fractals 134, 109696 (2020).

2 Goyal, M., Baskonus, H. M. \& Prakash, A. An efficient technique for a time fractional model of lassa hemorrhagic fever spreading in pregnant women. The European Physical Journal Plus 134, 482 (2019).

3 Khan, M. A., Atangana, Abdon. Modeling the dynamics of novel coronavirus (2019nCov) with fractional derivative. Alexandria Engineering Journal 59, 2379-2389 (2020).

4 Kumar, D., Singh, J., Al Qurashi, M. \& Baleanu, D. A new fractional SIRS-SI malaria disease model with application of vaccines, antimalarial drugs, and spraying. Advances in Difference Equations 2019, 278 (2019).

5 Lu, H., Stratton, C. W. \& Tang, Y. W. Outbreak of Pneumonia of Unknown Etiology in Wuhan China: the Mystery and the Miracle. Journal of Medical Virology 92, 401402 (2020).

6 Martcheva, M. An introduction to mathematical epidemiology. Vol. 61 9-31 (Springer, 2015).

7 Shah, K., Alqudah, M. A., Jarad, F. \& Abdeljawad, T. Semi-analytical study of Pine Wilt Disease model with convex rate under Caputo-Febrizio fractional order derivative. Chaos, Solitons \& Fractals 135, 109754 (2020).

8 Van den Driessche, P. \& Watmough, J. Reproduction numbers and sub-threshold endemic equilibria for compartmental models of disease transmission. Mathematical biosciences 180, 29-48 (2002).

9 Wu, J. T., Leung, K. \& Leung, G. M. Nowcasting and forecasting the potential domestic and international spread of the 2019-nCoV outbreak originating in Wuhan, China: a modelling study. The Lancet 395, 689-697 (2020). 
10 Read, J. M., Bridgen, J. R., Cummings, D. A., Ho, A. \& Jewell, C. P. Novel coronavirus 2019-nCoV: early estimation of epidemiological parameters and epidemic predictions. MedRxiv (2020).

11 Tang, B. et al. Estimation of the transmission risk of the 2019-nCoV and its implication for public health interventions. Journal of Clinical Medicine 9, 462 (2020).

12 Imai, N. et al. in Imperial College London (2020).

13 Zhu, H. et al. Host and infectivity prediction of Wuhan 2019 novel coronavirus using deep learning algorithm. BioRxiv (2020).

14 Cui, Q. et al. Dynamic variations of the COVID-19 disease at different quarantine strategies in Wuhan and mainland China. Journal of Infection and Public Health 13, 849-855 (2020).

15 Chen, T. et al. A mathematical model for simulating the transmission of Wuhan novel Coronavirus. bioRxiv (2020).

16 Hethcote, H. W. The mathematics of infectious diseases. SIAM review 42, 599-653 (2000).

17 Kucharski, A. J. et al. Early dynamics of transmission and control of COVID-19: a mathematical modelling study. The lancet infectious diseases 20, 553-558 (2020).

18 Mangoni, L. \& Pistilli, M. Epidemic analysis of Covid-19 in Italy by dynamical modelling. Available at SSRN 3567770 (2020).

19 Nadim, S. S., Ghosh, I. \& Chattopadhyay, J. Short-term predictions and prevention strategies for COVID-2019: A model based study. arXiv preprint arXiv:2003.08150 (2020).

20 Peng, L., Yang, W., Zhang, D., Zhuge, C. \& Hong, L. Epidemic analysis of COVID-19 in China by dynamical modeling. arXiv preprint arXiv:2002.06563 (2020).

21 Rabajante, J. F. Insights from early mathematical models of 2019-nCoV acute respiratory disease (COVID-19) dynamics. arXiv preprint arXiv:2002.05296 (2020). 
22 Wang, K. et al. Current trends and future prediction of novel coronavirus disease (COVID-19) epidemic in China: a dynamical modeling analysis. Mathematical biosciences and engineering 17, 3052 (2020).

23 Yang, C. \& Wang, J. A mathematical model for the novel coronavirus epidemic in Wuhan, China. Mathematical Biosciences and Engineering 17, 2708-2724 (2020).

24 Maier, B. F. \& Brockmann, D. Effective containment explains subexponential growth in recent confirmed COVID-19 cases in China. Science 368, 742-746 (2020).

25 Pal, D., Ghosh, D., Santra, P. \& Mahapatra, G. Mathematical Analysis of a COVID-19 Epidemic Model by using Data Driven Epidemiological Parameters of Diseases Spread in India. medRxiv (2020).

26 Tabassum, M. F., Saeed, M., Akgül, A., Farman, M. \& Chaudhry, N. A. Treatment of HIV/AIDS epidemic model with vertical transmission by using evolutionary Padéapproximation. Chaos, Solitons \& Fractals 134, 109686 (2020).

27 Farman, M., Saleem, M. U., Ahmed, M. \& Ahmad, A. Stability analysis and control of the glucose insulin glucagon system in humans. Chinese Journal of Physics 56, 13621369 (2018).

28 Gondim, J. A. \& Machado, L. Optimal quarantine strategies for the COVID-19 pandemic in a population with a discrete age structure. arXiv preprint arXiv:2005.09786 (2020).

29 Davies, N. G. et al. Age-dependent effects in the transmission and control of COVID19 epidemics. MedRxiv (2020).

30 Youssef, H., Alghamdi, N., Ezzat, M. A., El-Bary, A. A. \& Shawky, A. M. A Novel Mathematical Model (SEIRQ) of the COVID-19 Epidemic: Assessing the Epidemiological Rates of Diseases Spread in Saudi Arabia. (2020).

31 Jumpen, W., Wiwatanapataphee, B., Wu, Y. \& Tang, I. A SEIQR model for pandemic influenza and its parameter identification. International Journal of Pure and Applied Mathematics 52, 247-265 (2009). 
32 ud Din, R., Shah, K., Ahmad, I. \& Abdeljawad, T. Study of Transmission Dynamics of Novel COVID-19 by Using Mathematical Model. (2020).

33 Saudi Ministry of Health, <https://www.moh.gov.sa/en/Pages/default.aspx> (2020).

34 COVID-19 in Saudi_Arabia, <https://en.wikipedia.org/wiki/COVID19_pandemic_in_Saudi_Arabia> (2020).

35 Saudi Center for Diseases Prevention and Control, <https://covid19.cdc.gov.sa/ar/daily-updates-ar/> (2020). 
Figures

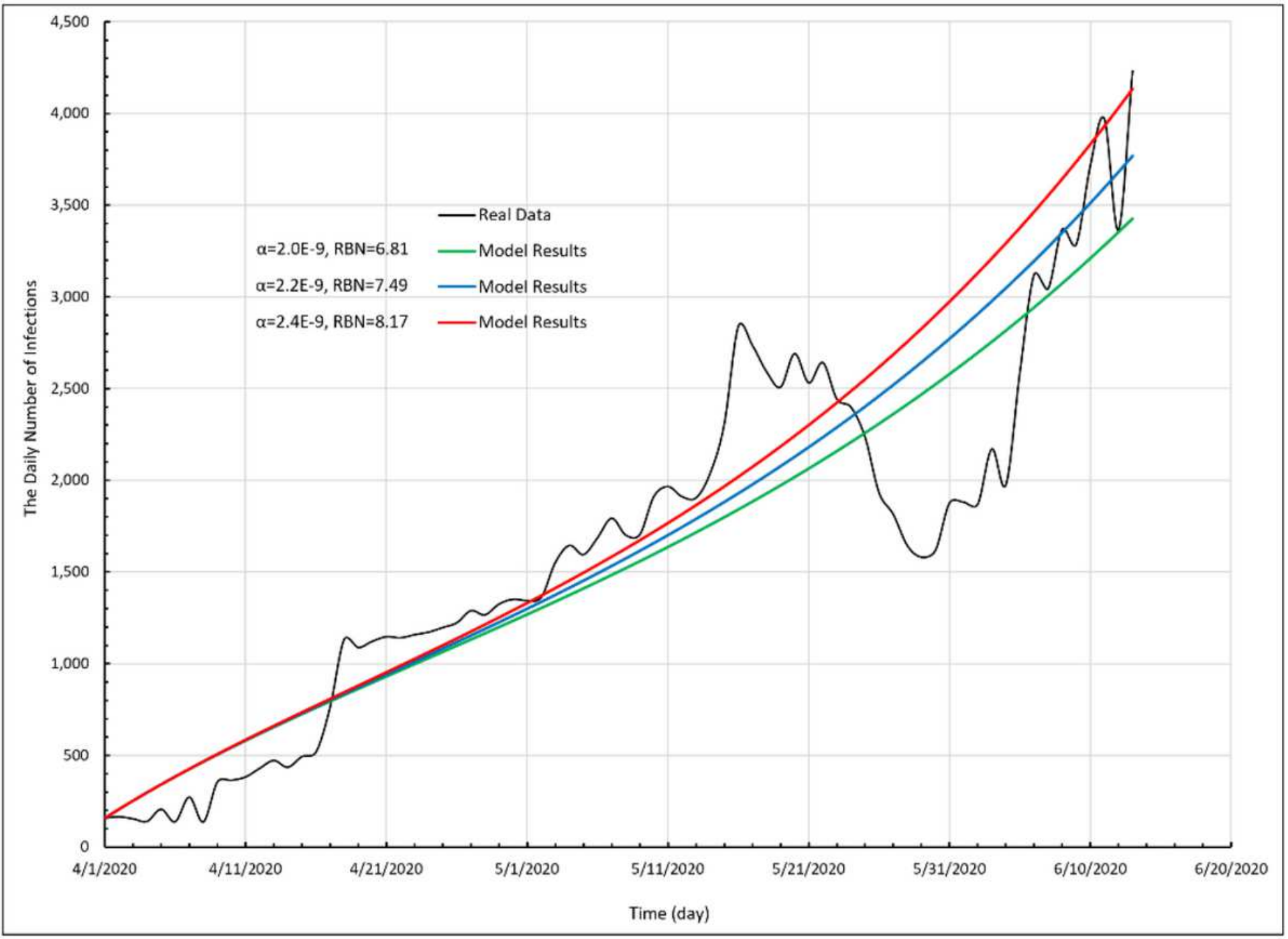

Figure 1

The number of daily infections based on the SEIQR model against the real data in Saudi Arabia between $4 / 1 / 2020$ and $6 / 13 / 2020$ 


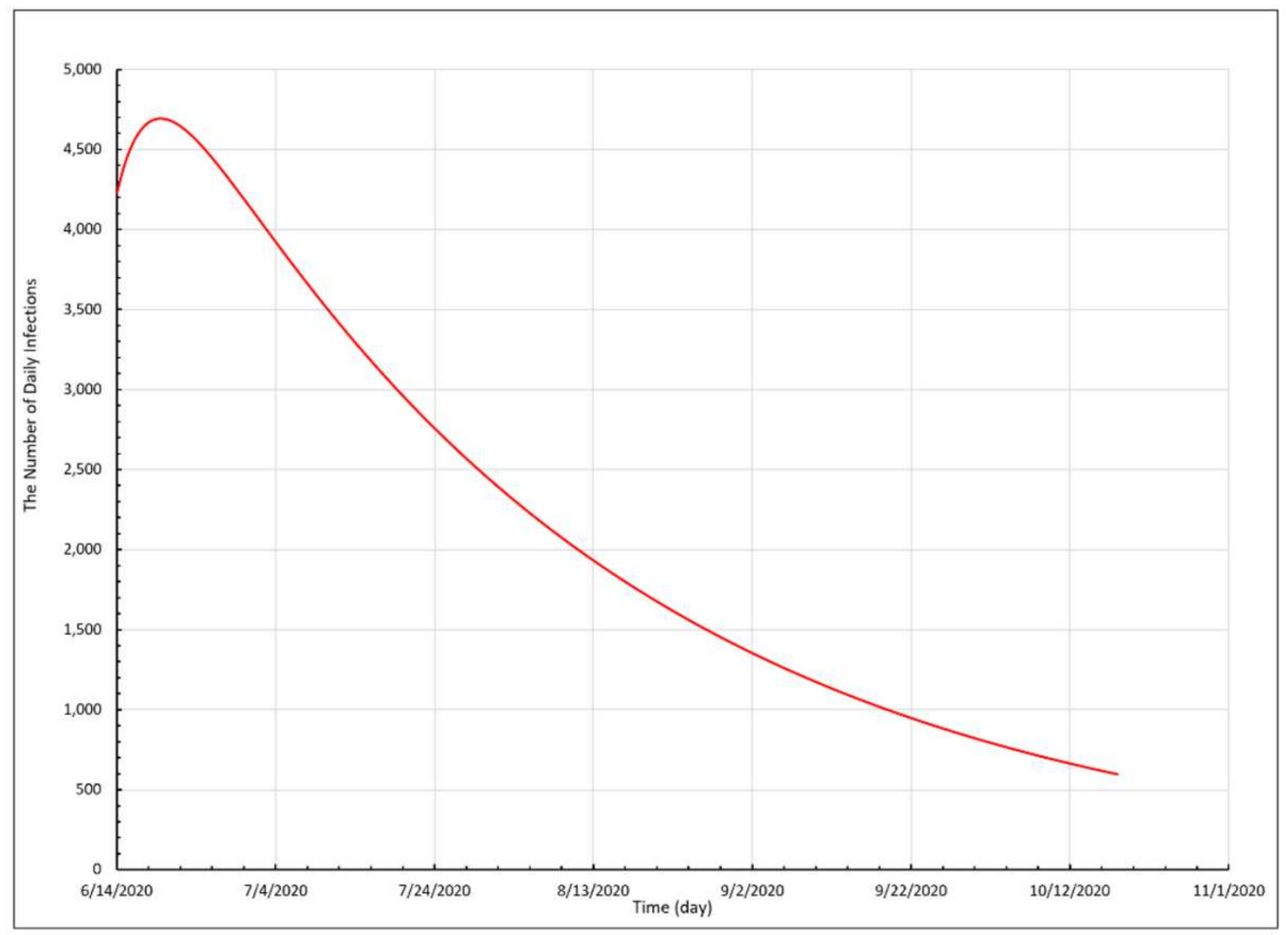

\section{Figure 2}

The number of daily infections based on the SEIQR model in Saudi Arabia was between 6/14/2020 and $10 / 18 / 2020$ if the values of the parameters, as in Table 3. 


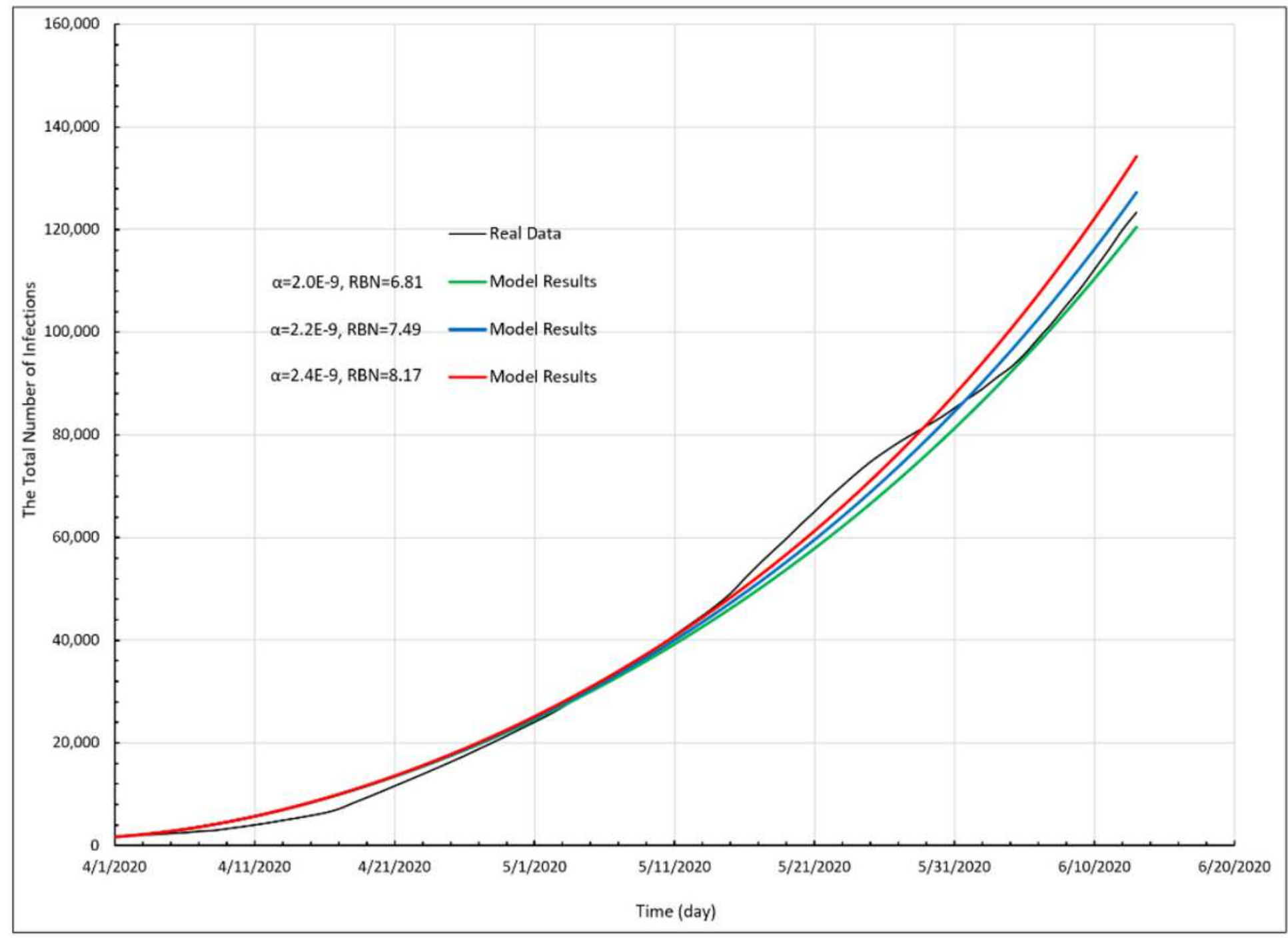

\section{Figure 3}

The total number of infections based on the SEIQR model against the real data in Saudi Arabia between $4 / 1 / 2020$ and $6 / 13 / 2020$ Now, 


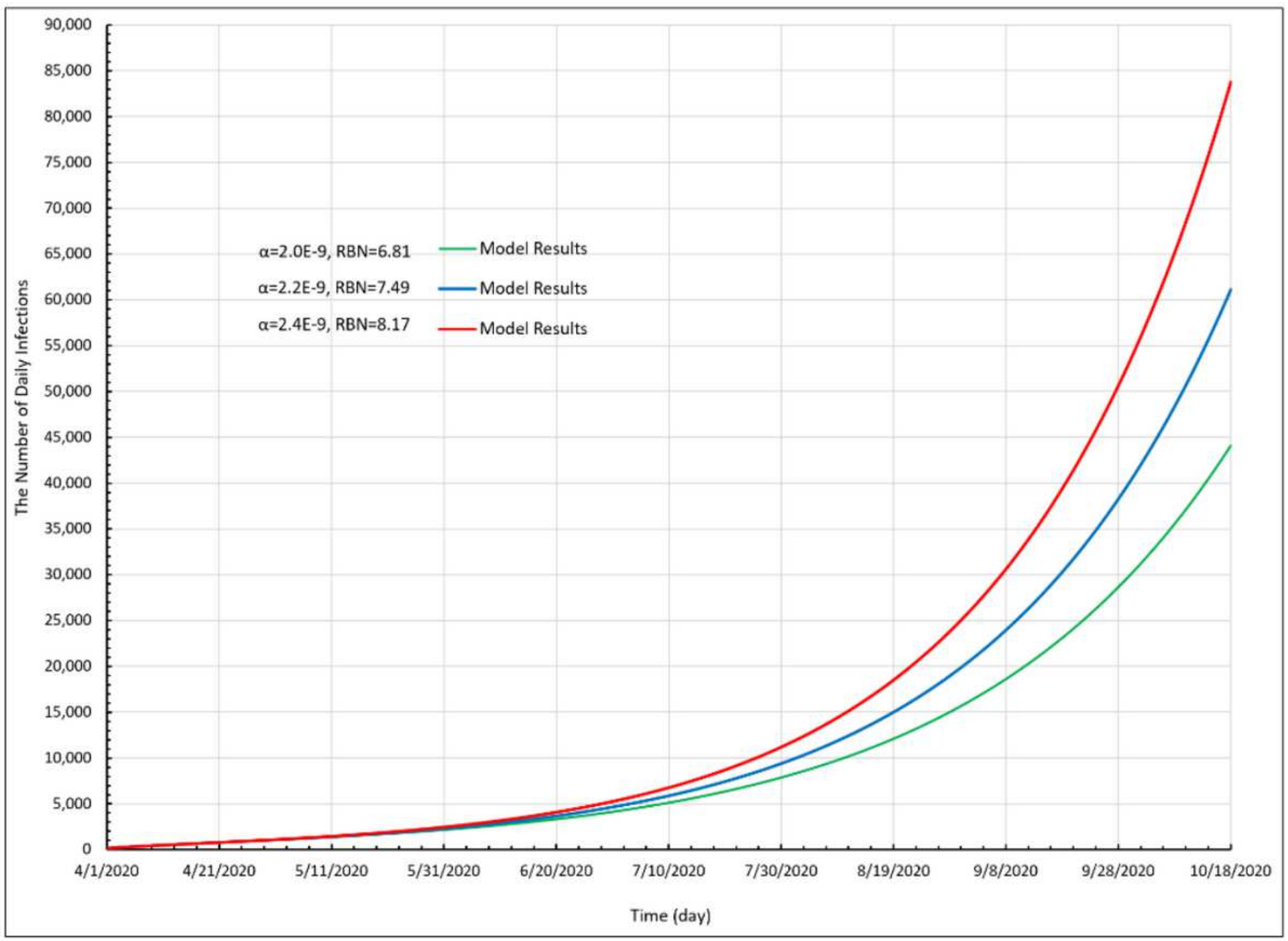

Figure 4

The number of daily infections based on the SEIQR model in Saudi Arabia between 4/1/2020 and $10 / 18 / 2020$ 


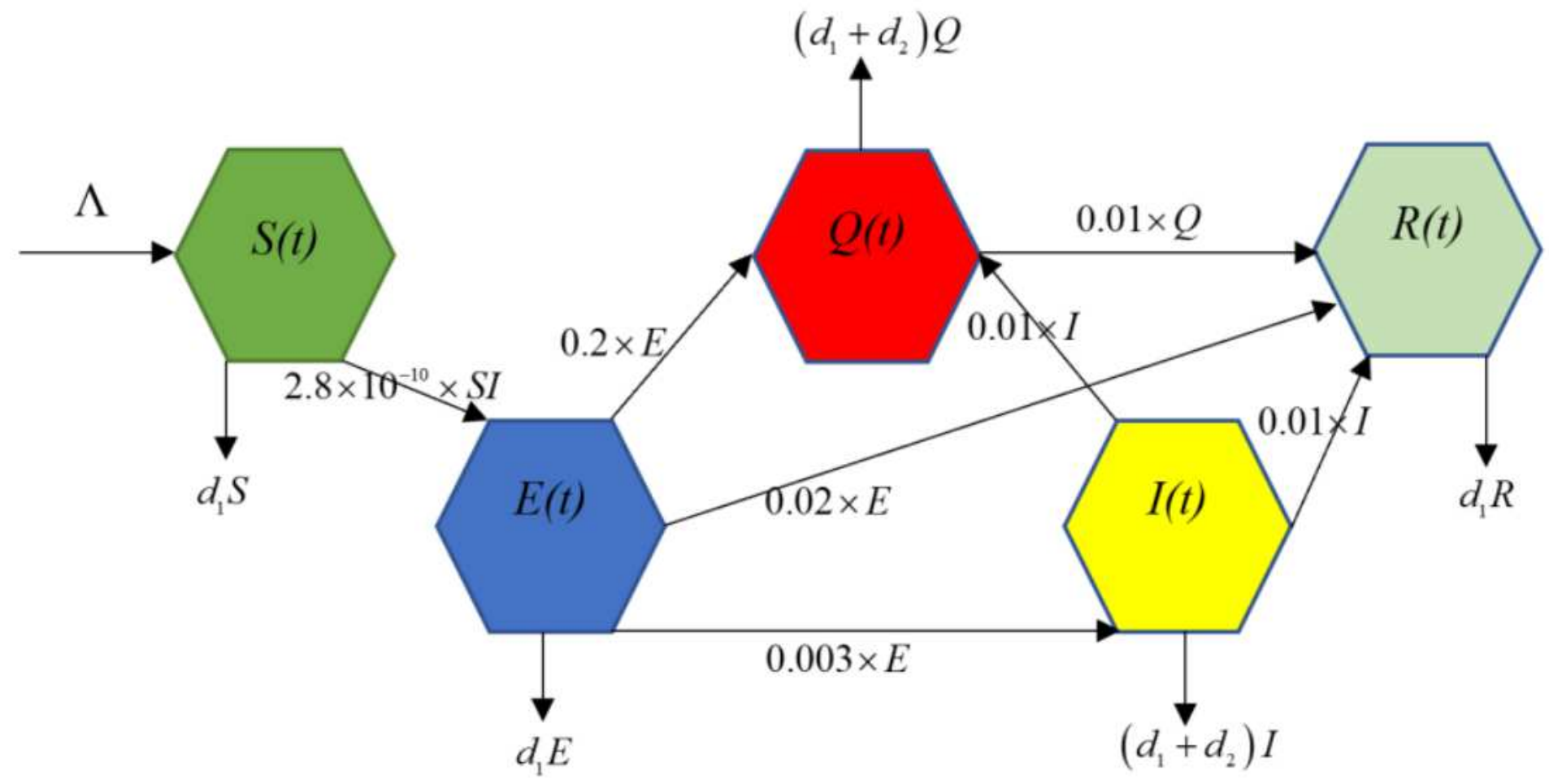

Figure 5

The flowchart of the ideal protocol based on the SEIQR model 


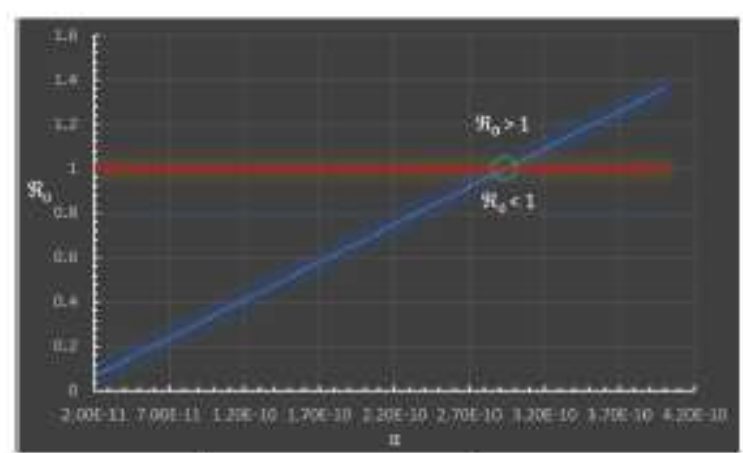

Figure $7 \mathrm{a} . \Re_{0}$ against $\alpha$

$\left(\alpha \leq 2.8 \times 10^{-10} \rightarrow \Re_{0} \leq 1\right)$

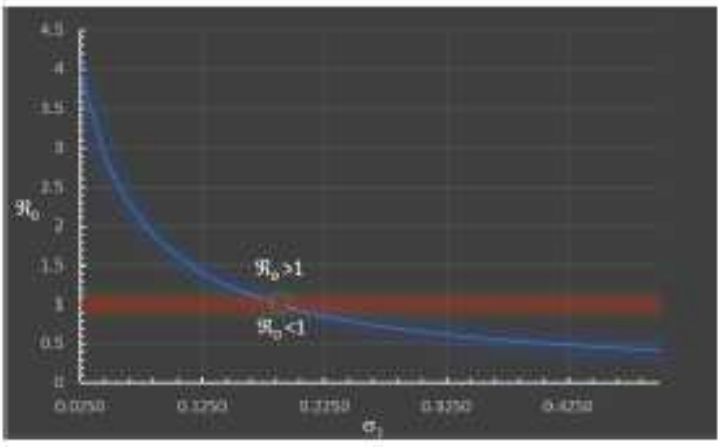

Figure $7 \mathrm{c} . \Re_{0}$ against $\sigma_{3}\left(\sigma_{3} \geq 0.18 \rightarrow \Re_{0} \leq 1\right)$

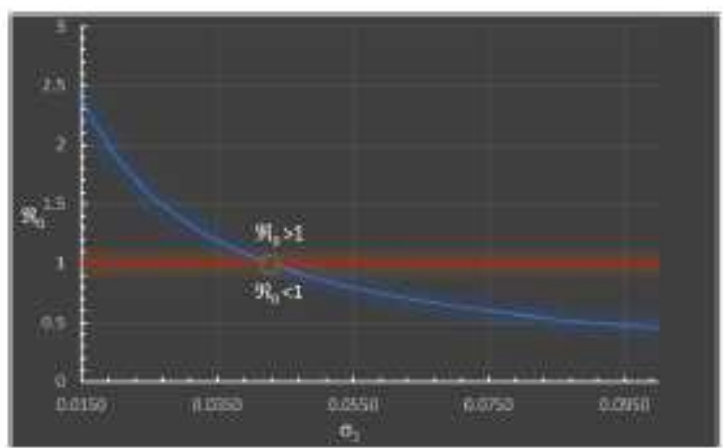

Figure 7e. $\Re_{0}$ against $\sigma_{2}\left(\sigma_{2} \geq 0.04 \rightarrow \Re_{0} \leq 1\right)$

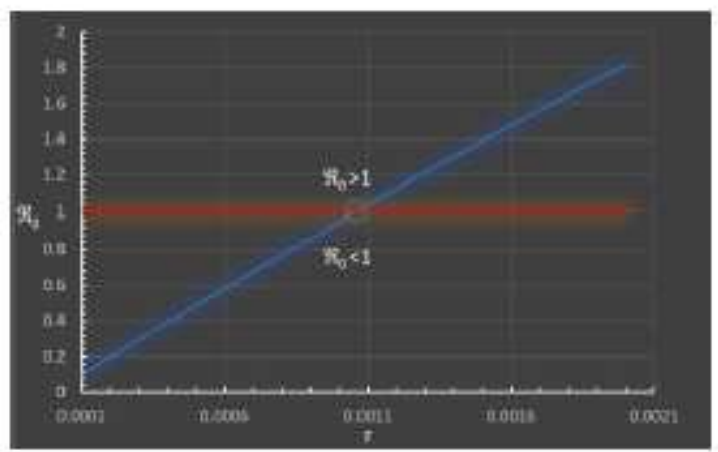

Figure $7 \mathrm{~b} . \Re_{0}$ against $r\left(r \leq 0.001 \rightarrow \Re_{0} \leq 1\right)$

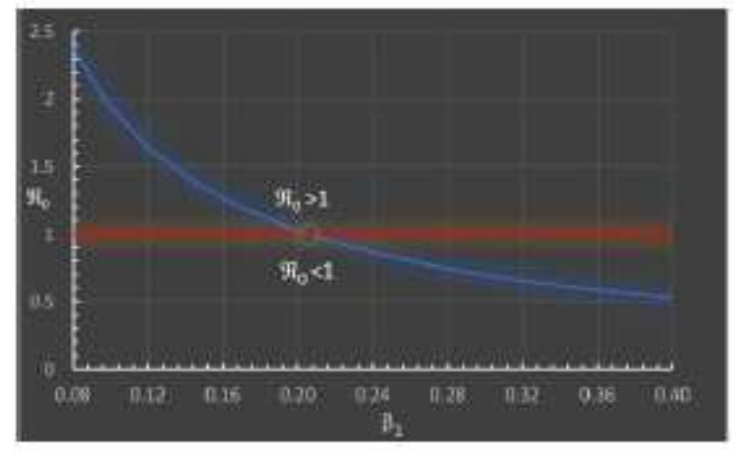

Figure $7 \mathrm{~d} . \Re_{0}$ against $\beta_{1}\left(\beta_{1} \geq 0.2 \rightarrow \Re_{0} \leq 1\right)$

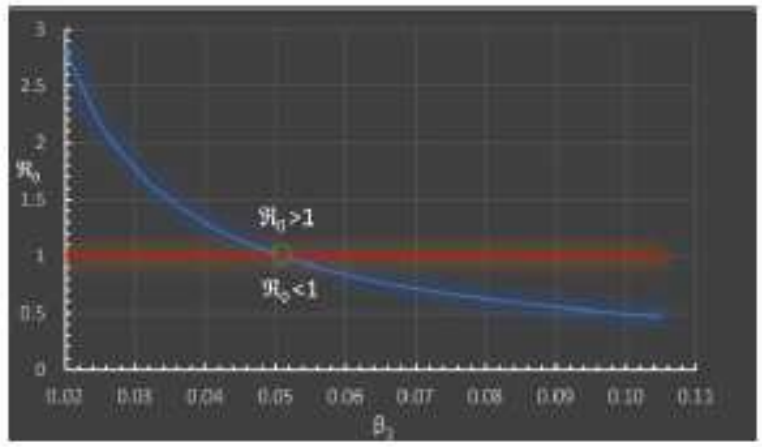

Figure 7f. $\Re_{0}$ against $\beta_{2}\left(\beta_{2} \geq 0.045 \rightarrow \Re_{0} \leq 1\right)$

\section{Figure 6}

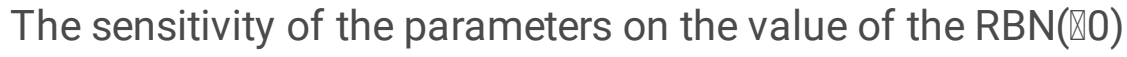




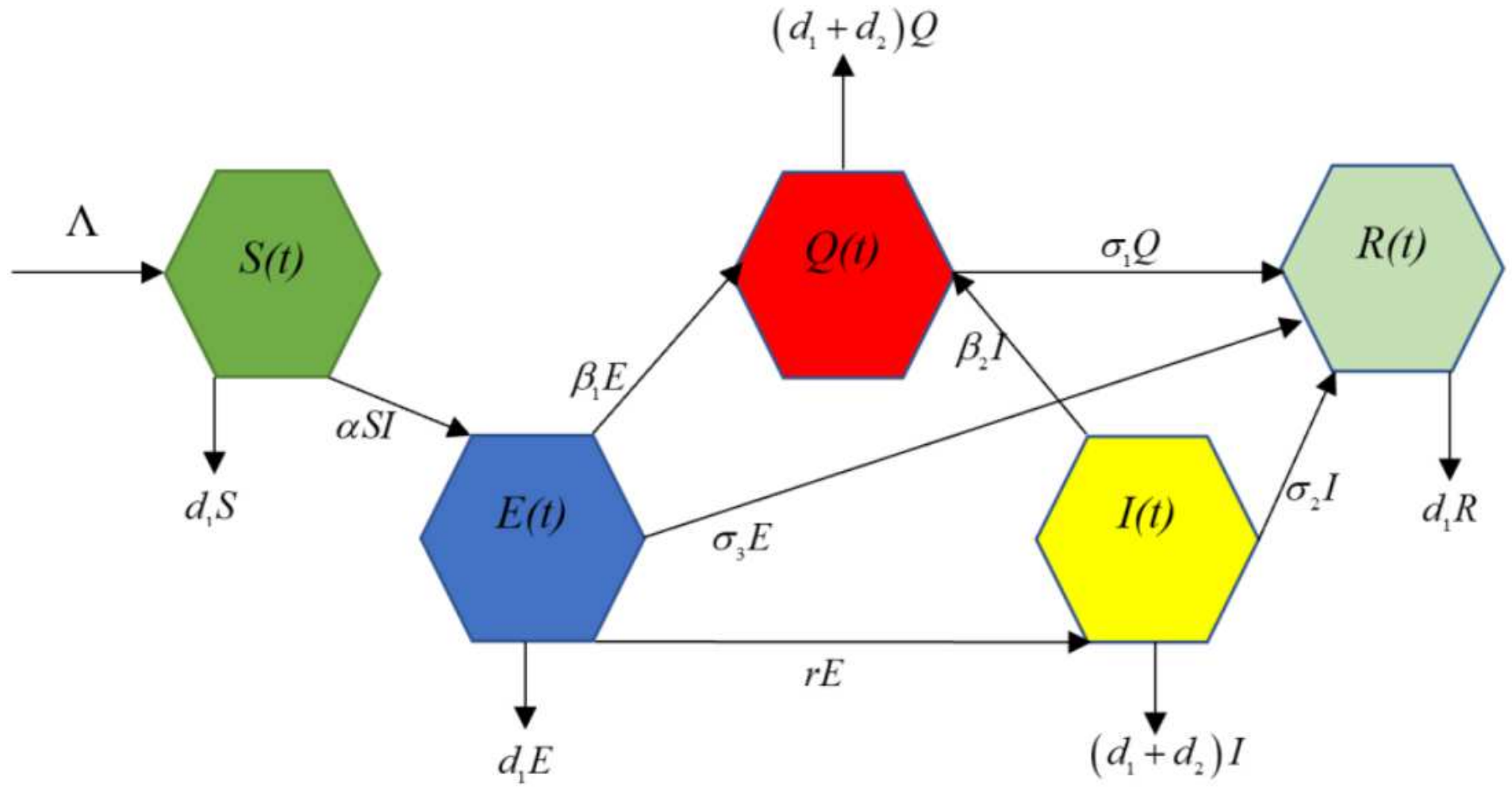

Figure 7

The flowchart of the SEIQR model 


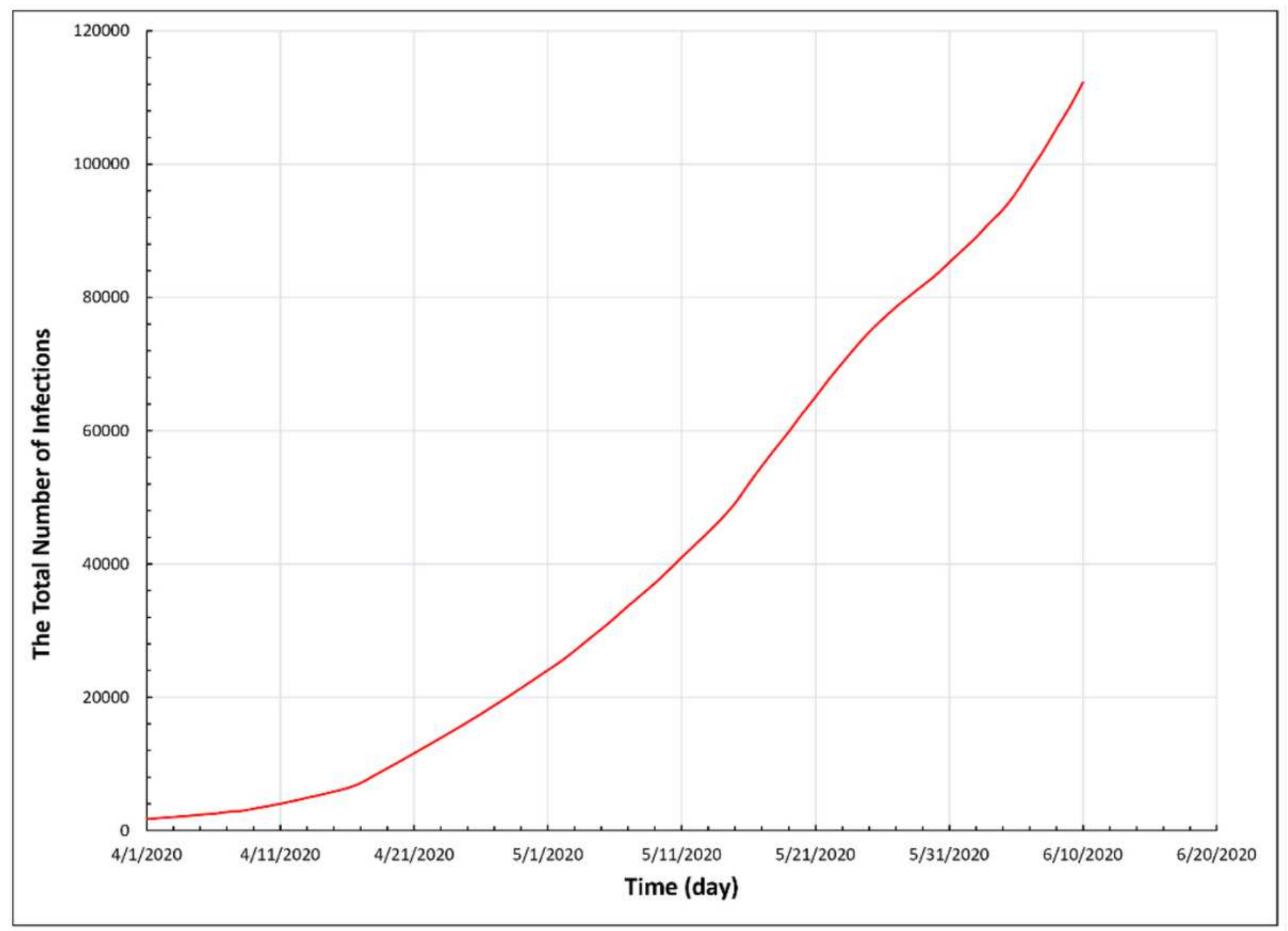

\section{Figure 8}

The total number of infections in Saudi Arabia between 4/1/2020 and 6/10/2020 


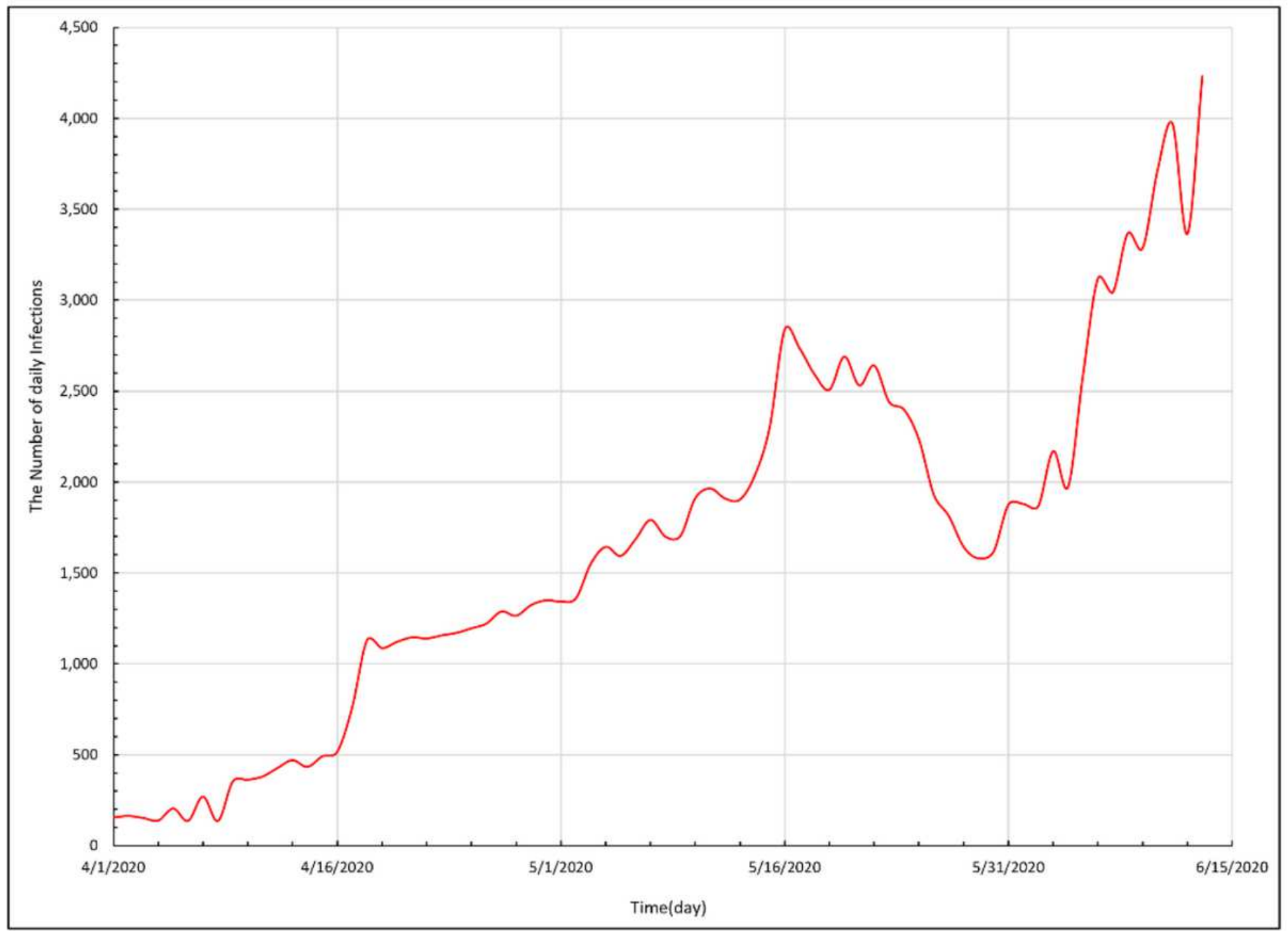

Figure 9

The real number of daily infections in Saudi Arabia between 4/1/2020 and 6/13/2020 